\title{
Substructural Identification of Flexural Rigidity for Beam-Like Structures
}

\author{
Ki-Young Koo ${ }^{1}$ and Jin-Hak $\mathbf{Y i}^{2,3}$ \\ ${ }^{1}$ College of Engineering, Mathematics and Physical Sciences, University of Exeter, Exeter, Devon EX4 4SB, UK \\ ${ }^{2}$ Coastal and Environmental Engineering Division, Korea Institute of Ocean Science and Technology, Ansan, \\ Gyeonggi 426-744, Republic of Korea \\ ${ }^{3}$ Department of Convergence Technology, Ocean Science and Technology School, Korea Maritime and Ocean University, \\ Busan 606-791, Republic of Korea \\ Correspondence should be addressed to Jin-Hak Yi; yijh@kiost.ac
}

Received 19 October 2014; Revised 23 December 2014; Accepted 30 December 2014

Academic Editor: Gyuhae Park

Copyright (C) 2015 K.-Y. Koo and J.-H. Yi. This is an open access article distributed under the Creative Commons Attribution License, which permits unrestricted use, distribution, and reproduction in any medium, provided the original work is properly cited.

\begin{abstract}
This study proposes a novel substructural identification method based on the Bernoulli-Euler beam theory with a single variable optimization scheme to estimate the flexural rigidity of a beam-like structure such as a bridge deck, which is one of the major structural integrity indices of a structure. In ordinary bridges, the boundary condition of a superstructure can be significantly altered by aging and environmental variations, and the actual boundary conditions are generally unknown or difficult to be estimated correctly. To efficiently bypass the problems related to boundary conditions, a substructural identification method is proposed to evaluate the flexural rigidity regardless of the actual boundary conditions by isolating an identification region within the internal substructure. The proposed method is very simple and effective as it utilizes the single variable optimization based on the transfer function formulated utilizing Bernoulli Euler beam theory for the inverse analysis to obtain the flexural rigidity. This novel method is also rigorously investigated by applying it for estimating the flexural rigidity of a simply supported beam model with different boundary conditions, a concrete plate-girder bridge model with different length of an internal substructure, a cantilever-type wind turbine tower structure with different type of excitation, and a steel box-girder bridge model with internal structural damages.
\end{abstract}

\section{Introduction}

For the optimal maintenance of civil infrastructures with a sufficient level of serviceability and safety, it is very important to evaluate major structural integrity indices and to monitor the changes of those values periodically [1-5]. In the case of ordinary bridges, structural integrity can be represented by several indices such as a remaining fatigue life, load carrying capacity, and natural frequencies. Among those, the load carrying capacity, which indicates the maximum allowable live load for a certain bridge, is the most useful index for decision on the structural integrity of a bridge and for bridge rating and maintenance as well. The load carrying capacity is related to many structural properties including flexural and torsional rigidities, deck mass, and boundary conditions as well. However, the flexural rigidity of a bridge deck is the most governing factor, and monitoring of flexural rigidity is essential for systematic and optimal bridge management systems. To evaluate the flexural rigidity of a bridge deck, several field testing methods can be carried out including a static loading test and a dynamic vehicle test. However, for a reliable evaluation, it is necessary to model accurately the boundary conditions at the interfaces between a bridge deck and supporting structural members such as abutments and bridge piers by considering the current deteriorated status.

In cases of existing bridges in service, the supporting structural members may not behave according to the designed supporting conditions even though they were fabricated and installed as typical bearings such as rollers and hinges due to aging and other environmental changes [6-8]. For example, rollers can behave similarly to fixed shoes due to aging and deterioration, and this can reduce the vertical deflections by partially constraining the rotational deflection at the boundaries under vehicle loads, and therefore the load 
carrying capacity can be overestimated when the boundary conditions are not correctly reflected. In this study, a new concept for a substructural identification method is proposed incorporating a single variable optimization scheme for the flexural rigidity estimation of a beam-like structure such as a bridge deck without considering boundary conditions and also without carrying out complex and complicated experimental modal analysis. The applicability of the proposed method is verified through numerical simulation and also model tests for a simply supported beam model and a steel box-girder bridge model.

The substructural identification method can efficiently reduce the measuring points and identification parameters by isolating the estimation region within an internal substructure, and hence the instability during the identification process, which is a kind of inverse analyses, can be significantly reduced. This approach has been developed by many researchers over the last two decades. Oreta and Tanabe [9] proposed a Kalman filter-based substructural identification for estimating structural damages, and Yun and Lee [10] proposed a substructural identification using the ARMAX model in a time domain to identify damages in frame structures. Yun and Lee utilized the sequential prediction error method while Oreta and Tanabe incorporated Kalman filtering. While they utilized time domain substructural identification methods, Koh et al. [11] proposed an iterative method combining substructural identification and progressive identification in frequency domain, and Koh and Shankar [12] proposed a substructural identification method that does not require interface measurements to avoid problems related to measurement at the interfaces. More recently, Zhang et al. [13] extended Koh and Shankar's method for applications to arbitrary excitation cases to reduce computation time and increase identification accuracy by introducing an exponential window method and they investigated the proposed method with a 7-level small-scale steel frame model. Li et al. [14] also proposed a substructural identification based on the response reconstruction in frequency-domain model updating, and the method could identify the substructural parameters by reducing the measured acceleration and reconstructed responses obtained from the updated model. Li and Law [15] developed the substructural damage identification method to apply the moving load excitation cases, and they verified their scheme with numerical simulation tests. Weng et al. [16, 17] proposed the substructural identification using substructural flexibility matrix for damage detection and model updating as well, and they applied the proposed substructural flexibility matrix-based method successfully to identify the damages and structural parameters of a 3-story small-scale portal frame structure and also a large-scale $600 \mathrm{~m}$ tall Guangzhou New TV tower. Even though substructural identification techniques are consistently being studied and further developed for the successful real applications, several issues are still under development. One of the main issues in substructural identification is related to the reliable measurement on the interface region between internal and external substructures, especially rotational responses. In the case of shear-building models, the issue related to measure the rotational degree of freedom can be bypassed, and some researchers resolved the unmeasured rotational degree of freedom responses by adopting the mode expansion technique or model reduction technique. The latter techniques such as model expansion and reduction rely on the reliable reference model, of course; therefore the direct measurement can be considered owing to the recent development on sensor technology and the high-performance dynamic inclinometers can be much more feasible in the near future. It is notable that the substructural identification methods need to carry out repeated tests to narrow down the damaged region. If the failure critical members can be predetermined through numerical analyses and/or preliminary structural tests, the internal substructure to be monitored can be more reasonably assigned and the proposed method can be more efficiently applied. And also the damaged locations can be known by means of other nondestructive testing methods; the damage severity can be more accurately assessed by the proposed substructural identification method.

In this study, a novel substructural identification method is proposed to estimate the flexural rigidity of a beamlike structure using a single variable optimization scheme based on the transfer function formulated utilizing Bernoulli Euler beam theory and relatively simple measurement setups with measuring the rotational responses by introducing two sensors near the interface regions. And the performance of the proposed method is also rigorously investigated through experimental and numerical example studies with a simply supported beam model with different boundary condition, a concrete plate-girder bridge model with different substructural length, a cantilever-type wind turbine tower model with different type of excitation force, and a steel box-girder bridge model with internal structural damages. The identification performance is evaluated in the view point whether it can identify the flexural rigidity of a beam-like structure with different and unknown boundary conditions and how much the length of an internal substructure and the type of excitation force affect the estimation results.

\section{Formulation of Substructural Identification Using Single Variable Optimization}

2.1. Layout of Substructural Identification. Flexural rigidity of a beam-like structure is the most important structural parameter determining the load carrying capacity of a structure. It is also a very simple and intuitive parameter used for evaluating the deterioration level due to structural damages and aging. For identification of the flexural rigidity of a bridge, the boundary conditions at the connecting parts with abutments and bridge piers need to be carefully considered since the structural responses are related with not only the flexural rigidity but also the boundary conditions which are apt to change due to temperature and humidity variations as well as aging and deterioration. However, it is generally very difficult to examine the concurrent status of the boundary conditions in operation.

Hence, a new concept for the substructural identification technique is proposed based on the single variable optimization, and this approach is very easy and simple to implement 


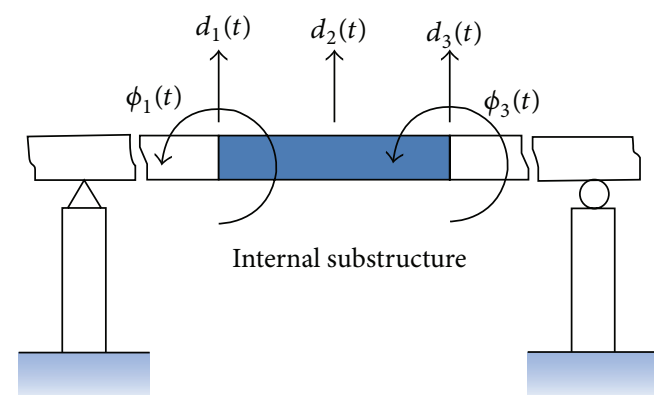

(a) Internal substructure of a bridge deck

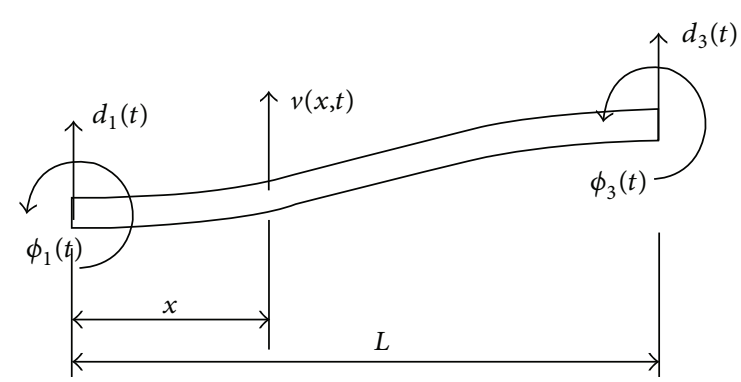

(b) Input-output relationship of internal substructure

FIGURE 1: Substructural system of a bridge for estimating flexural rigidity.

to an existing structural health monitoring system by utilizing the divide and conquer strategy especially for complex civil infrastructures with numerous structural members.

The fundamental concept of the substructural identification is summarized in Figure 1. First, an internal substructure of interest can be selected for identification and then the vertical accelerations at both interfaces, $\ddot{d}_{1}(t)$ and $\ddot{d}_{3}(t)$; and the rotational accelerations at the same positions, $\ddot{\phi}_{1}(t)$ and $\ddot{\phi}_{3}(t)$, are measured simultaneously with the vertical acceleration at the center of the internal substructure, $\ddot{d}_{2}(t)$. It should be noted that $\ddot{d}_{2}(t)$ is a function of the input excitations $\ddot{d}_{1}(t), \ddot{\phi}_{1}(t), \ddot{d}_{3}(t)$, and $\ddot{\phi}_{3}(t)$ and the dynamic properties of the substructure including flexural rigidity can be estimated by identification based on the input and output measurements.

The transfer function $H(\omega)$ between the input responses at the boundaries and the output responses at the internal substructure is derived from the Bernoulli-Euler beam theory in Section 2.1 and the flexural rigidity estimation procedure is formulated based on the single variable optimization in Section 2.2.

2.2. Transfer Function of Substructure. The dynamic governing equation of the Bernoulli-Euler beam is represented as follows:

$$
\left(E I v^{\prime \prime}\right)^{\prime \prime}+\rho A \ddot{v}=0
$$

where $E I, \rho$, and $A$ are the flexural rigidity, the mass density, and the sectional area of the beam, respectively, and dots denote derivatives with respect to $t$ and primes derivatives with respect to $x$. By using separation of variables, $v(x, t)=$ $X(x) T(t)$, the above partial differential equation can be transformed into a set of two ordinary differential equations (ODEs) ((3) and (4)) with respect to $X(x)$ and $T(t)$ as follows:

$$
\begin{gathered}
\frac{X^{(4)}}{X}=-\frac{\rho A}{E I} \frac{\ddot{T}}{T}=\lambda^{4}, \\
X^{(4)}-\lambda^{4} X=0, \\
\ddot{T}+\omega^{2} T=0,
\end{gathered}
$$

where $\lambda$ and $\omega$ are arbitrary constants with the relation of $\lambda^{4}=$ $\rho A \omega^{2} /(E I)$. The general solutions of a set of two ODEs in (3) and (4) are obtained as

$$
\begin{gathered}
X(x)=C_{1} \sinh (\lambda x)+C_{2} \cosh (\lambda x)+C_{3} \sin (\lambda x) \\
+C_{4} \cos (\lambda x) \\
T(t)=B e^{i \omega t},
\end{gathered}
$$

where $C_{i}$ 's and $B$ are arbitrary constants. Hence the general solution of the governing equation in (1) can be derived as in (6) by combining $X(x)$ and $T(t)$ in (5)

$$
\begin{aligned}
v(x, t) & =X(x) T(t) \\
& =\left[\begin{array}{llll}
\sinh (\lambda x) & \cosh (\lambda x) & \sin (\lambda x) & \cos (\lambda x)
\end{array}\right] \mathbf{C} e^{i \kappa t},
\end{aligned}
$$

where $\mathbf{C}=\left[\begin{array}{llll}C_{1} & C_{2} & C_{3} & C_{4}\end{array}\right]^{T}$ is an arbitrary coefficient column vector.

The particular solution can be obtained considering the time varying boundary conditions at the interfacial locations as follows:

$$
\begin{aligned}
v(0, t) & =d_{1}(t), & v(L, t)=d_{3}(t), \\
\left.\frac{\partial v(x, t)}{\partial x}\right|_{x=0}=\phi_{1}(t), & & \left.\frac{\partial v(x, t)}{\partial x}\right|_{x=L}=\phi_{3}(t) .
\end{aligned}
$$

The above boundary condition constitutes the following linear algebraic equation:

$$
\begin{aligned}
& {\left[\begin{array}{cccc}
0 & 1 & 0 & 1 \\
\lambda & 0 & \lambda & 0 \\
\sinh \lambda L & \cosh \lambda L & \sin \lambda L & \cos \lambda L \\
\lambda \cosh \lambda L & \lambda \sinh \lambda L & \lambda \cos \lambda L & -\lambda \sin \lambda L
\end{array}\right] \mathrm{C} e^{i \kappa t}} \\
& =\left\{\begin{array}{l}
d_{1}(t) \\
\phi_{1}(t) \\
d_{3}(t) \\
\phi_{3}(t)
\end{array}\right\} .
\end{aligned}
$$




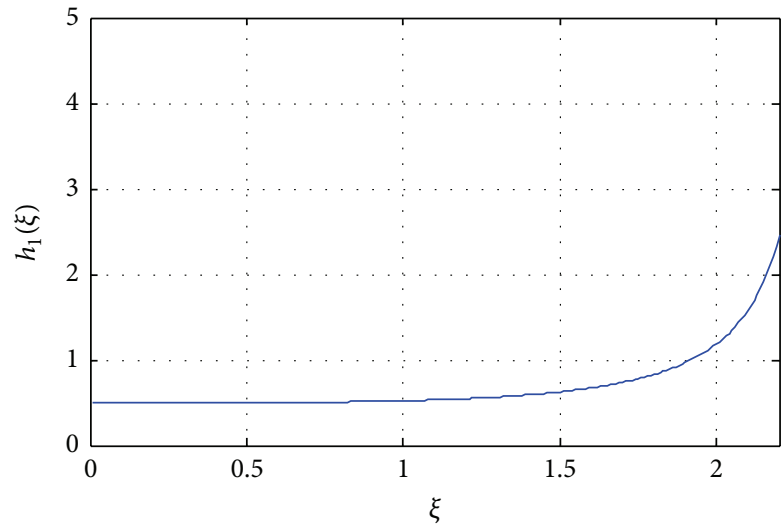

(a) $\bar{h}_{1}(\xi)$

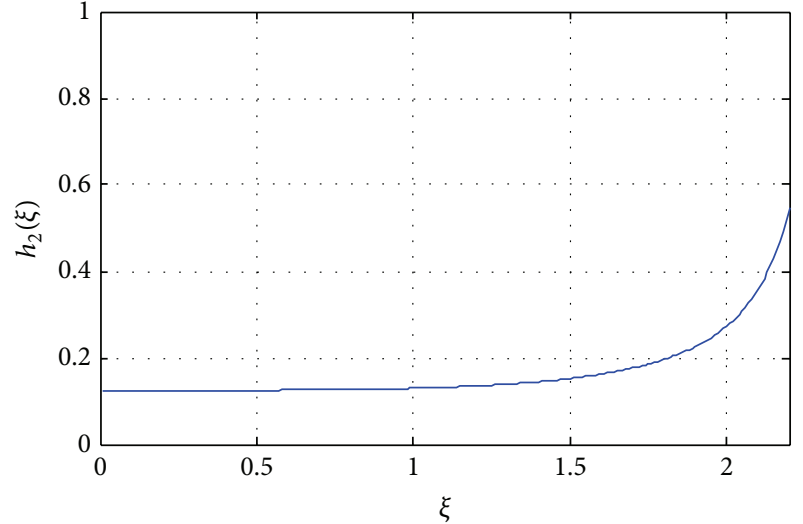

(b) $\bar{h}_{2}(\xi)$

FIGURE 2: Transfer functions between interfacial and internal responses of the internal substructure.

When we set the matrix in left-hand side as $\mathbf{A}$ and the vector in right-hand side as $\mathbf{u}(t)$ as follows

$$
\begin{gathered}
\mathbf{A} \triangleq\left[\begin{array}{cccc}
0 & 1 & 0 & 1 \\
\lambda & 0 & \lambda & 0 \\
\sinh \lambda L & \cosh \lambda L & \sin \lambda L & \cos \lambda L \\
\lambda \cosh \lambda L & \lambda \sinh \lambda L & \lambda \cos \lambda L & -\lambda \sin \lambda L
\end{array}\right], \\
\mathbf{u}(t) \triangleq\left[\begin{array}{llll}
d_{1}(t) & \phi_{1}(t) & d_{3}(t) & \phi_{3}(t)
\end{array}\right]^{T}
\end{gathered}
$$

then the particular solution can be obtained as (10) by exchanging the coefficient column vector $\mathrm{C}^{i \omega t}$ in (6) with $\mathbf{A}^{-1} \mathbf{u}(t)$ in (8):

$$
v(x, t)=\left[\begin{array}{lll}
\sinh \lambda x & \cosh \lambda x \sin \lambda x \cos \lambda x
\end{array}\right] \mathbf{A}^{-1} \mathbf{u}(t) .
$$

And the transfer function $\mathbf{H}(x, \omega)$ of $v(x, t)$ with respect to the input displacements $\mathbf{u}(t)$ at both boundaries can be obtained by applying Fourier transform to both sides of (10) as follows:

$$
\begin{aligned}
& F\{V(x, t)\}
\end{aligned}
$$

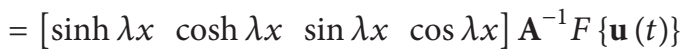

$$
\begin{aligned}
& =\mathbf{H}(x, \omega) F\{\mathbf{u}(t)\}, \\
& \therefore \mathbf{H}(x, \omega)=\left[\begin{array}{lll}
\sinh \lambda x & \cosh \lambda x & \sin \lambda x \cos \lambda x
\end{array}\right] \mathbf{A}^{-1} \text {. }
\end{aligned}
$$

The transfer function for $x=L / 2$ (at the center of internal substructure) can be derived as

$$
\mathbf{H}(\omega)_{x=L / 2}=\left[\begin{array}{llll}
h_{1}(\omega) & h_{2}(\omega) & h_{3}(\omega) & h_{4}(\omega)
\end{array}\right],
$$

where $h_{i}(\omega)$ 's are

$$
\begin{aligned}
h_{1}(\omega)= & h_{3}(\omega) \\
= & \frac{1}{2} \frac{\sin (\lambda L / 2)+\sinh (\lambda L / 2)}{\sin (\lambda L / 2) \cosh (\lambda L / 2)+\cos (\lambda L / 2) \sinh (\lambda L / 2)}, \\
h_{2}(\omega)= & -h_{4}(\omega)=-\frac{1}{2 \lambda L} \\
& \cdot \frac{\cos (\lambda L / 2)-\cosh (\lambda L / 2)}{\sin (\lambda L / 2) \cosh (\lambda L / 2)+\cos (\lambda L / 2) \sinh (\lambda L / 2)} .
\end{aligned}
$$

The transfer functions in (12) can be further simplified using the nondimensional variable $\xi$ as

$$
\mathbf{H}(\xi)=\left[\begin{array}{llll}
\bar{h}_{1}(\xi) & L \bar{h}_{2}(\xi) & \bar{h}_{3}(\xi) & L \bar{h}_{4}(\xi)
\end{array}\right]
$$

where

$$
\begin{gathered}
\xi=\frac{\lambda L}{2}=\sqrt[4]{\frac{\rho A}{E I}} \frac{\sqrt{\omega} L}{2}, \\
\bar{h}_{1}(\xi)=\bar{h}_{3}(\xi)=\frac{1}{2} \frac{\sin \xi+\sinh \xi}{\sin \xi \cosh \xi+\cos \xi \sinh \xi} \\
\bar{h}_{2}(\xi)=-\bar{h}_{4}(\xi)=-\frac{1}{4 \xi} \frac{\cos \xi-\cosh \xi}{\sin \xi \cosh \xi+\cos \xi \sinh \xi} .
\end{gathered}
$$

The transfer functions $\bar{h}_{1}(\xi)$ and $\bar{h}_{2}(\xi)$ with respect to the nondimensional variable $\xi$ are plotted in Figure 2 and it can be easily observed that the transfer function values are positive and monotonically increased as the variable $\xi$ increases. And it can also be observed that the transfer function values are very flat in the range of 0 to 1.5 , which means that the small errors in this region can arouse a significant level of estimation errors due to the insensitiveness. From (17), it can be also observed that the transfer function values are not available (i.e., divergent) and there is a discontinuity point when the denominator approaches to zero like $\tan \xi+\tanh \xi=$ 0 .

2.3. Estimation of Flexural Rigidity. The input and output relationship between the responses at the interfaces and at the center of the internal substructure can be represented as

$$
\begin{aligned}
& d_{2}(\omega)=\mathbf{H}(\xi)\left[\begin{array}{llll}
d_{1}(\omega) & \phi_{1}(\omega) & d_{3}(\omega) & \phi_{3}(\omega)
\end{array}\right]^{T} \\
& =\bar{h}_{1}(\xi) d_{1}(\omega)+\bar{h}_{2}(\xi) L \phi_{1}(\omega)+\bar{h}_{3}(\xi) d_{3}(\omega) \\
& +\bar{h}_{4}(\xi) L \phi_{3}(\omega) .
\end{aligned}
$$


By using the relationships $\bar{h}_{3}(\xi)=\bar{h}_{1}(\xi)$ and $\bar{h}_{4}(\xi)=-\bar{h}_{2}(\xi)$, the equation above can be simplified as a two inputs and one output system as follows:

$$
\begin{aligned}
d_{2}(\omega) & =\bar{h}_{1}(\xi)\left(d_{1}(\omega)+d_{3}(\omega)\right)+\bar{h}_{2}(\xi) L\left(\phi_{1}(\omega)-\phi_{3}(\omega)\right) \\
& =\bar{h}_{1}(\xi) u_{1}(\omega)+\bar{h}_{2}(\xi) L u_{2}(\omega)
\end{aligned}
$$

where $u_{1}(\omega) \triangleq d_{1}(\omega)+d_{3}(\omega)$ and $u_{2}(\omega) \triangleq \phi_{1}(\omega)-\phi_{3}(\omega)$. By multiplying $\lim _{T \rightarrow \infty}(1 / T) d_{2}^{*}(\omega)$ on both sides of (19), a representation of spectral densities can be obtained as follows for convenience of the calculation and interpretation of the terms:

$$
\begin{array}{r}
\lim _{T \rightarrow \infty} \frac{1}{T} d_{2}^{*}(\omega) d_{2}(\omega)=\bar{h}_{1}(\xi) \lim _{T \rightarrow \infty} \frac{1}{T} d_{2}^{*}(\omega) u_{1}(\omega) \\
+\bar{h}_{2}(\xi) L \lim _{T \rightarrow \infty} \frac{1}{T} d_{2}^{*}(\omega) u_{2}(\omega) \\
S_{y y}(\omega)=\bar{h}_{1}(\xi) S_{1 y}(\omega)+\bar{h}_{2}(\xi) L S_{2 y}(\omega)
\end{array}
$$

The power spectral density functions $S_{y y}(\omega), S_{1 y}(\omega)$, and $S_{2 y}(\omega)$ are auto- and cross-PSD functions defined as follows and they can be easily obtained using the measured four input responses at both interfaces and the one output response at the center of the internal substructure:

$$
\begin{aligned}
& S_{y y}(\omega) \triangleq \lim _{T \rightarrow \infty} \frac{1}{T} d_{2}^{*}(\omega) d_{2}(\omega), \\
& S_{1 y}(\omega) \triangleq \lim _{T \rightarrow \infty} \frac{1}{T} d_{2}^{*}(\omega) u_{1}(\omega), \\
& S_{2 y}(\omega) \triangleq \lim _{T \rightarrow \infty} \frac{1}{T} d_{2}^{*}(\omega) u_{2}(\omega) .
\end{aligned}
$$

Finally, the unknown parameter $\xi$ can be obtained by minimizing the error norm of (21) as follows:

$$
\begin{aligned}
& \min J(\xi) \\
& =\int_{\Omega}\left\{S_{y y}(\omega)-\left(\bar{h}_{1}(\xi) S_{1 y}(\omega)+\bar{h}_{2}(\xi) L S_{2 y}(\omega)\right)\right\}^{2} d \Omega,
\end{aligned}
$$

where $\Omega$ is the integral domain, that is, the frequency range to be used for the identification. Even though there is no limitation how to determine the integral domain among all the frequency ranges, a frequency range around the first natural frequency $\left(f_{1}\right)$ is preferable. First of all, the level of structural responses is certainly larger than the level of measurement noise signals around the resonant frequency regions. Secondly, the mode shape of the first mode fits with the assumption used for formulation in this study. The Bernoulli-Euler beam theory assumes that the flexural deflection is dominant by ignoring the shear deformation, and this assumption requires that the ratio of the effective length $\left(L_{e}\right)$ over the height of the beam is greater than 10 ;

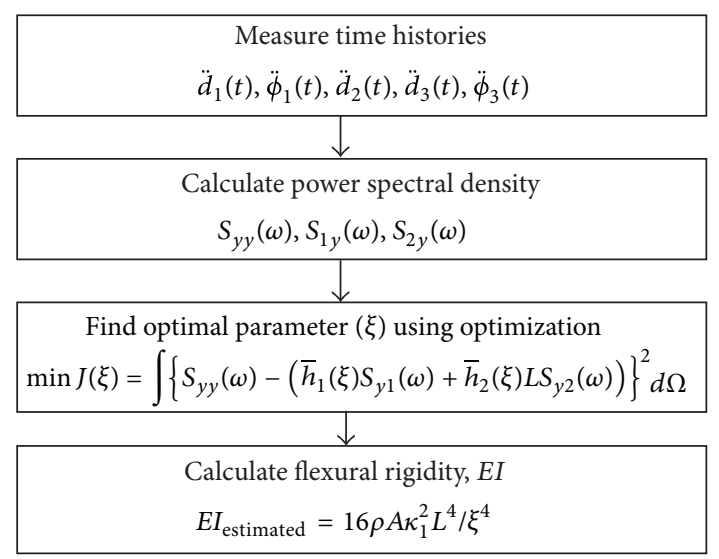

FIgURE 3: Procedure for flexural rigidity estimation using substructural identification.

that is, $L / h \gg 10$. For the several lower modes for a simply supported beam, the effective length can be calculated as $L_{e}=$ $L_{0}, L_{e}=L_{0} / 2$, and $L_{e}=L_{0} / 3$ for the 1st, 2 nd, and $3 \mathrm{rd}$ modes, respectively, and $L_{0}$ represents the span length of a beam.

Equation (23) is a single variable optimization problem, and the unknown variable $\xi$ can be solved by any kind of optimization procedure such as a steepest-decent method. Using the optimal solution $\xi_{\text {opt }}$, the flexural rigidity $E I$ can be estimated as follows based on (15):

$$
E I_{\text {estimated }}=\frac{16 \rho A \omega_{1}^{2} L^{4}}{\xi_{\mathrm{opt}}^{4}} \quad\left(\omega_{1}=2 \pi f_{1}\right) .
$$

The proposed algorithm for estimating flexural rigidity using 4 responses at the interfaces and 1 response in the internal substructure is summarized as shown in Figure 3.

\section{Verification Study}

3.1. Simple Beam Structure with Different Boundary Conditions. The proposed method can estimate the flexural rigidity of a bridge deck without considering the boundary conditions by incorporating the substructural identification. To validate the applicability, the test model with sectional dimension of $100 \mathrm{~mm} \times 6 \mathrm{~mm}$ (Figure 4), which can change the boundary conditions easily to fixed, hinged, roller, and friction, is designed and dynamic tests are carried out with different boundary conditions. The substructure is set as the internal $1.4 \mathrm{~m}$ part among the beam with a $2 \mathrm{~m}$ length, and the two vertical accelerations $\left(A_{1}\right.$ and $A_{2}$ and $A_{4}$ and $A_{5}$ in Figure $4(\mathrm{~b}))$ at the interface parts and the vertical acceleration $\left(A_{3}\right.$ in Figure $\left.4(b)\right)$ at the center of the substructure are measured. The vertical vibration at the interface is obtained using the mean of the adjacent two accelerations $\left(\therefore \ddot{d}_{1}(t)=\right.$ $\left.\left(A_{1}+A_{2}\right) / 2, \ddot{d}_{3}(t)=\left(A_{4}+A_{5}\right) / 2\right)$ and the rotational acceleration is obtained by dividing the difference of the adjacent two accelerations with the distance between the two sensors $\left(L_{\text {dist }}=5 \mathrm{~cm}\right)\left(\therefore \ddot{\phi}_{1}(t)=\left(A_{1}-A_{2}\right) / L_{\text {dist }}, \ddot{\phi}_{3}(t)=\right.$ $\left.\left(A_{4}-A_{5}\right) / L_{\text {dist }}\right)$.

Relaxation tests, which can be carried out by slightly pushing and quickly releasing the beam, are performed 


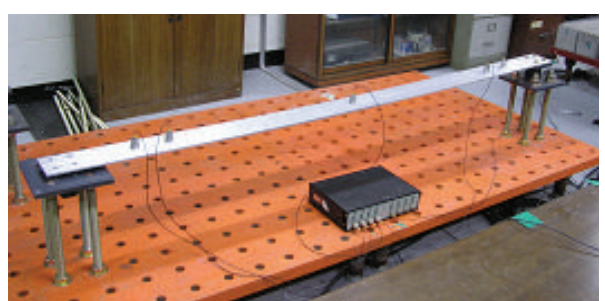

(a) Overview

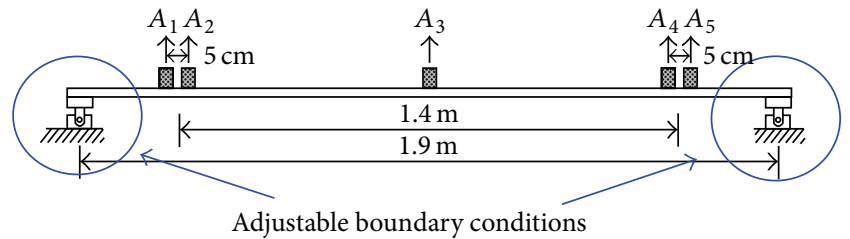

(b) Sensor configuration

FIgURE 4: Simple beam test structure.

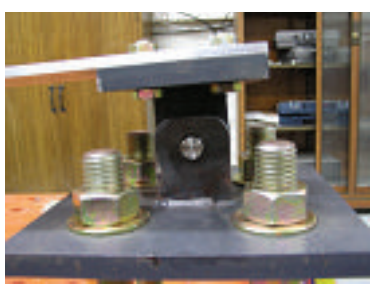

(a) Hinge condition

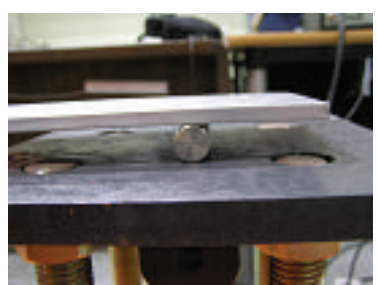

(b) Roller condition

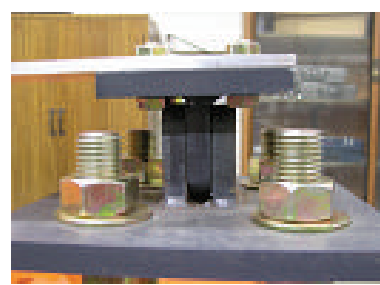

(c) Fixed condition

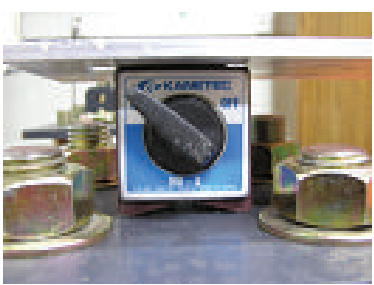

(d) Friction condition

FIGURE 5: Different boundary conditions.
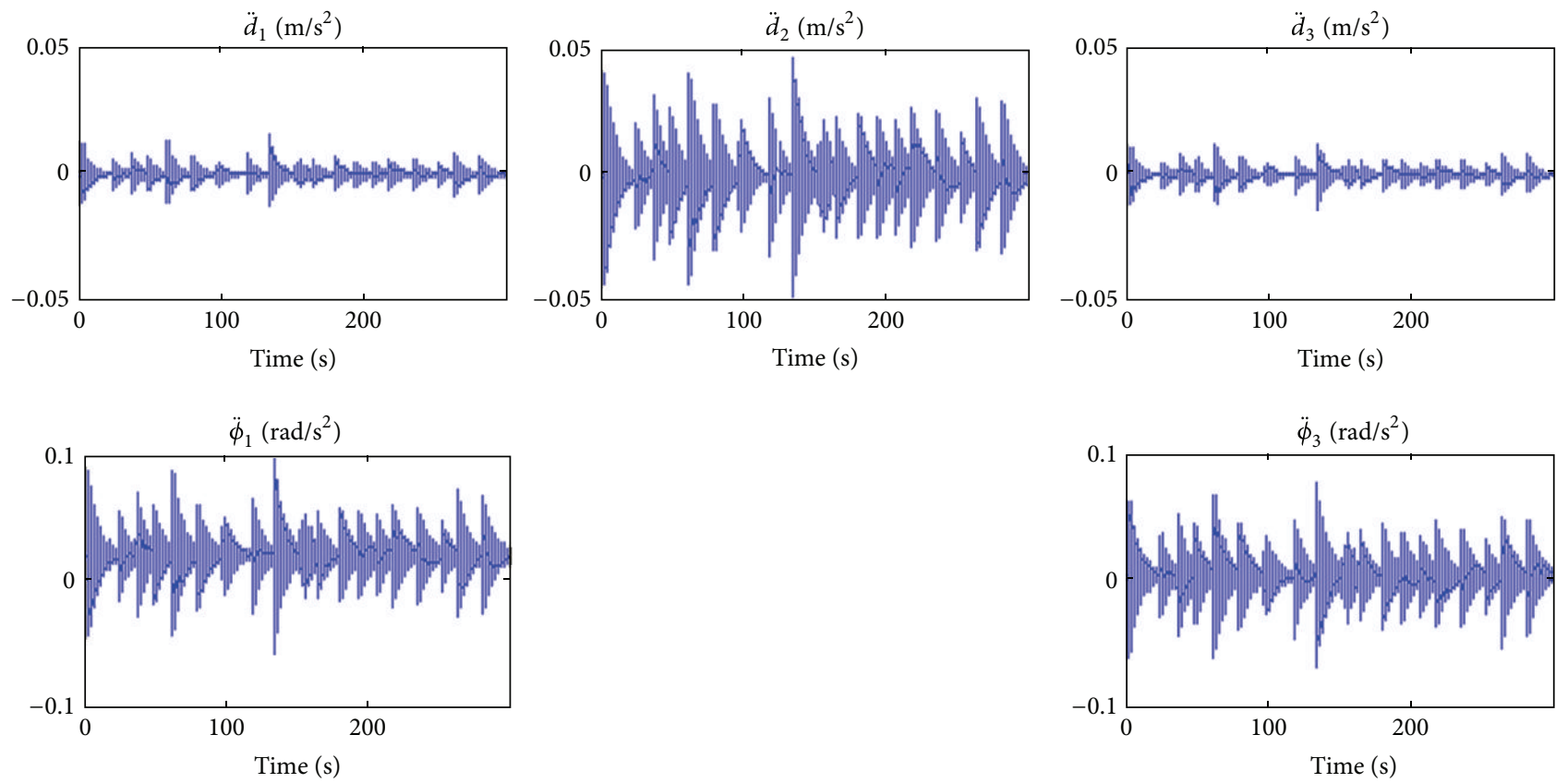

FIgURE 6: Input and output data for Case 1.

for the 4 different boundary conditions shown in Figure 5 and Table 1. Figure 6 shows the approximated responses at the interfaces $\left(\ddot{d}_{1}(t), \ddot{\phi}_{1}(t), \ddot{d}_{3}(t)\right.$, and $\left.\ddot{\phi}_{3}(t)\right)$ and the output response at the internal substructure $\left(\ddot{d}_{2}(t)\right)$ for the corresponding Case 1. Figure 7 represents the PSD function of $\ddot{d}_{2}(t)$.

Finally, the nondimensional variable $\xi$ is obtained by optimizing the PSD relationship of the substructural system around the first resonant frequency. The flexural rigidity $(E I)$ is then estimated using (24). The estimated values
TABLE 1: Test scenarios.

\begin{tabular}{lcccc}
\hline Boundary conditions & 1 & 2 & 3 & 4 \\
\hline Left end & Fixed & Roller & Roller & Friction \\
Right end & Fixed & Fixed & Hinged & Hinged \\
\hline
\end{tabular}

are summarized as shown in Table 2. From the results, we can observe that the first natural frequency of the system with different boundary conditions is changed from $8.179 \mathrm{~Hz}$ for Case 1 (fixed-fixed) to $5.981 \mathrm{~Hz}$ for Case 2 (roller-fixed), 
TABLE 2: Estimated flexural rigidity (exact value: $128.2 \mathrm{Nm}$ ).

\begin{tabular}{lcccc}
\hline Cases & $f_{1}(\mathrm{~Hz})$ & $\xi$ & $E I(\mathrm{Nm})$ & Error $(\%)$ \\
\hline 1 & 8.179 & 1.718 & 122.0 & -4.8 \\
2 & 5.981 & 1.443 & 131.1 & 2.2 \\
3 & 3.845 & 1.673 & 124.0 & -3.2 \\
4 & 4.883 & 1.308 & 129.6 & 1.0 \\
\hline
\end{tabular}

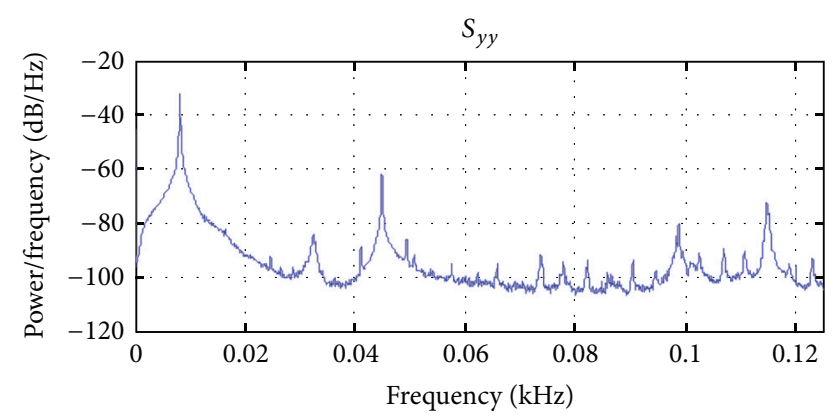

FIGURE 7: PSD function of output response at the internal substructure for Case 1 .

$3.845 \mathrm{~Hz}$ for Case 3 (roller-hinged), and $4.883 \mathrm{~Hz}$ for Case 4 (friction-hinged) according to the boundary conditions. Even though the first natural frequency of substructure varies significantly, the flexural rigidity is very precisely and accurately estimated by the proposed method. The maximum and minimum values are $131.1 \mathrm{Nm}$ for Case 2 and $122.0 \mathrm{Nm}$ for Case 1, and the relative difference between maximum and minimum estimates of estimated values is about $7.2 \%$. The relative errors of the estimates with respect to the exact value $(128.2 \mathrm{Nm})$ are $-4.8 \% \sim 2.2 \%$. The minimal amount of errors may be due to the approximation for obtaining the rotational acceleration using the difference of the acceleration measurements at the adjacent two points. It can be concluded that the proposed method can estimate flexural rigidity even when the boundary conditions are not fully understood.

\subsection{Application to Concrete Plate-Girder Bridge Model. From} the study described in Section 3.1, it is found that the proposed method can be successfully applied to estimate the flexural rigidity of a beam without considering an actual boundary condition. In this section, the effect of the length of an internal substructure is investigated using numerically simulated data for a simple beam model as shown in Figure 8. This simple beam is assumed to be supported by a roller and hinge at each end. Numerical simulation is performed using the commercial structural analysis program SAP2000, and band limited white noise is used for the input excitation as a form of low amplitude ambient vibration of ground (i.e., microtremor). The mass density $(\rho)$ and Young's modulus $(E)$ of concrete are assumed to be $2402.8 \mathrm{~kg} / \mathrm{m}^{3}$ and $24.82 \mathrm{GPa}$, respectively, and the area $(A)$ and the 2 nd moment of inertia $(I)$ of the section are considered as $0.2 \mathrm{~m}^{2}$ and $6.6667 \times$ $10^{-4} \mathrm{~m}^{4}$, respectively.

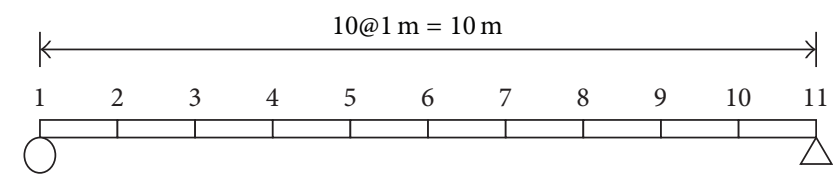

FIGURE 8: The concrete plate-girder bridge model (10 $\mathrm{m}=10 @ 1 \mathrm{~m})$.

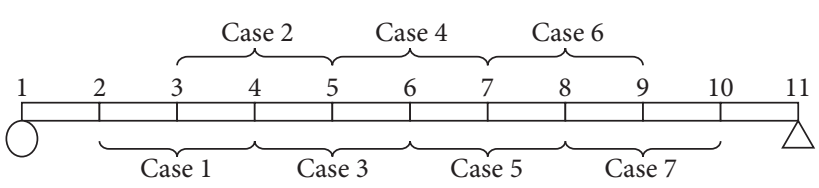

(a) 7 cases with substructure length of $2 \mathrm{~m}$

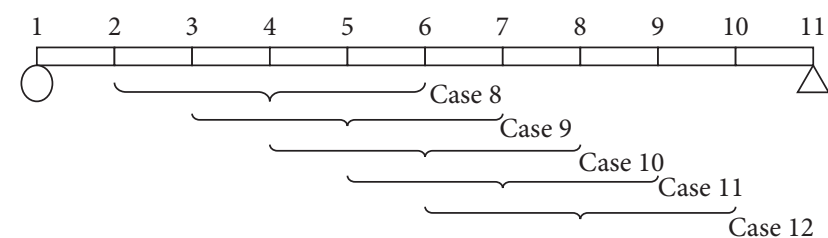

(b) 5 cases with substructure length of $4 \mathrm{~m}$

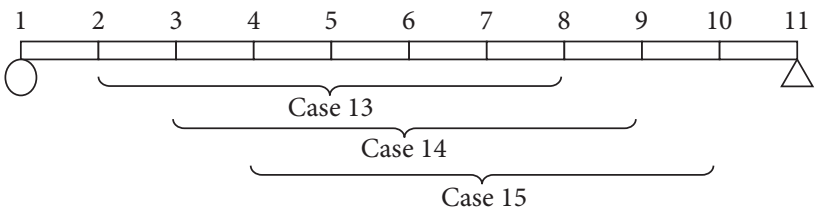

(c) 3 cases with substructure length of $6 \mathrm{~m}$

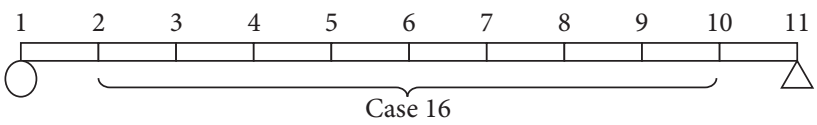

(d) 1 case with substructure length of $8 \mathrm{~m}$

FIGURE 9: 16 different cases for the substructural system.

In this study, the effect of the length of an internal substructure on the estimation accuracy is intensively investigated using 16 different substructural cases with 4 different lengths as shown in Figure 9. For each case, the vertical and rotational accelerations at both interfacial nodes are obtained using the numerical simulation results. And the flexural rigidity is estimated from the calculated acceleration responses.

Figure 10 shows the input and output acceleration responses at both interfaces and at the center of the internal substructure for the Substructural Case 1. Figure 11 shows the PSD function, $S_{y y}(\omega)$, of the vertical acceleration at the center of the internal substructure, $\ddot{d}_{2}(t)$, the cross spectral density functions, $S_{1 y}(\omega)$ and $S_{2 y}(\omega)$, of the sum of vertical accelerations at both ends, $\ddot{d}_{1}(t)+\ddot{d}_{3}(t)$, and the difference between rotational accelerations at both ends, $-\ddot{\phi}_{1}(t)+\ddot{\phi}_{3}(t)$, with respect to the vertical acceleration at the center of the internal substructure, respectively.

From the estimation results in Figure 12, it can be easily observed that the estimation error can be significantly 


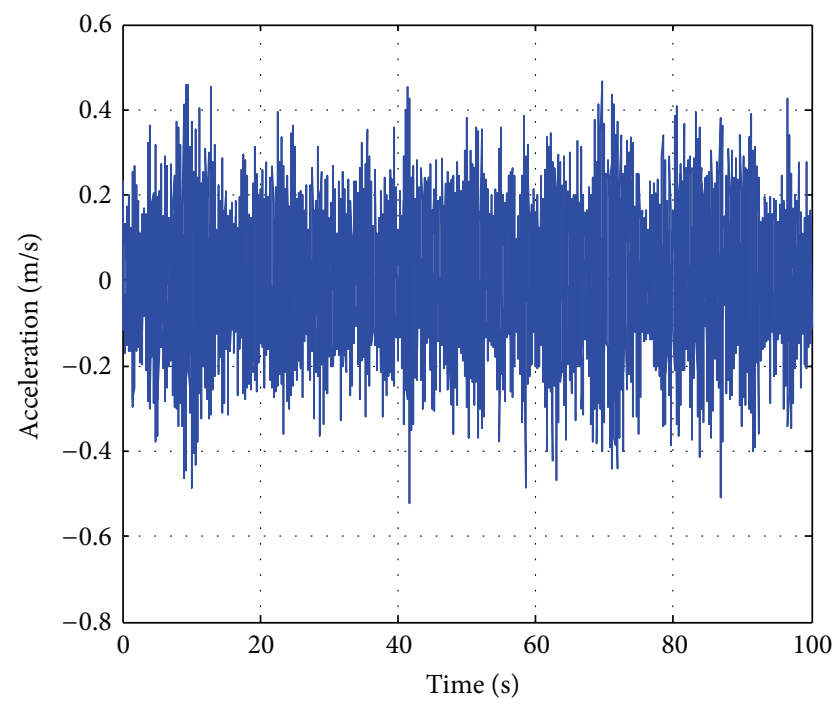

(a) $\ddot{u}_{1}(t)=\ddot{d}_{1}(t)+\ddot{d}_{3}(t)$

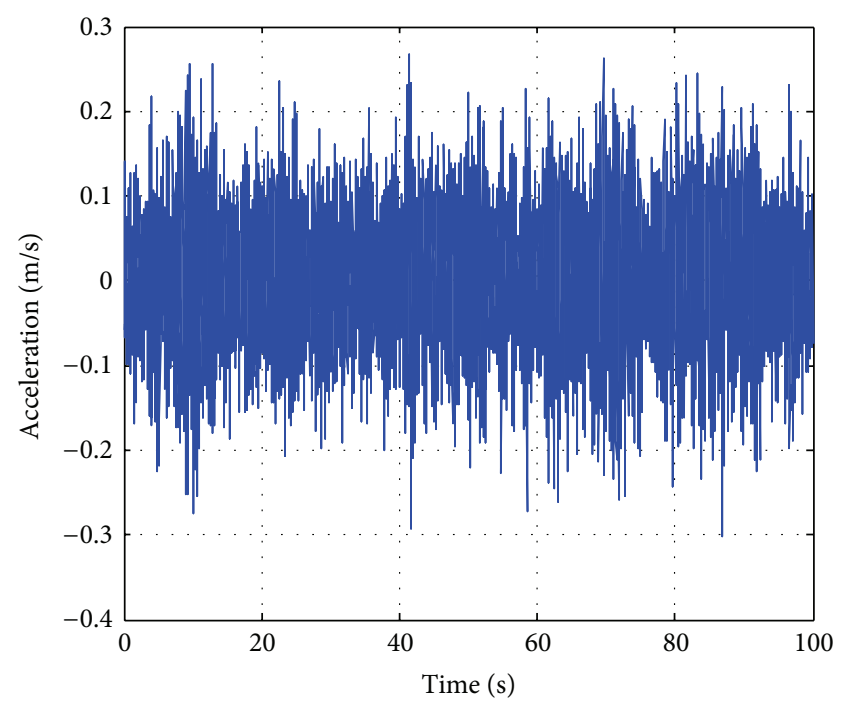

(b) $\ddot{d}_{2}(t)$

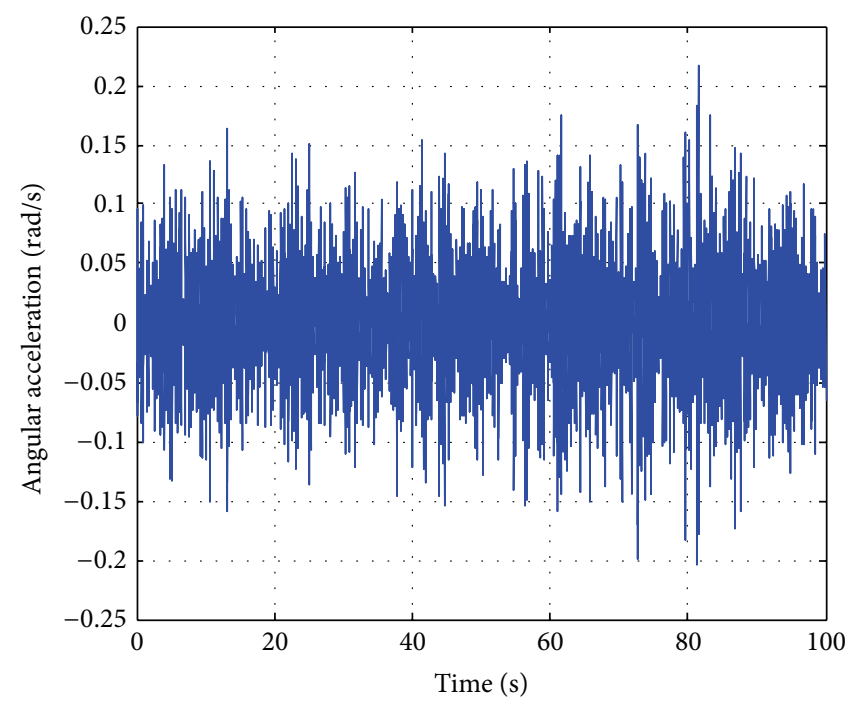

(c) $\ddot{u}_{2}(t)=\ddot{\phi}_{1}(t)-\ddot{\phi}_{3}(t)$

FIGURE 10: Input and Output Data for Case 1.

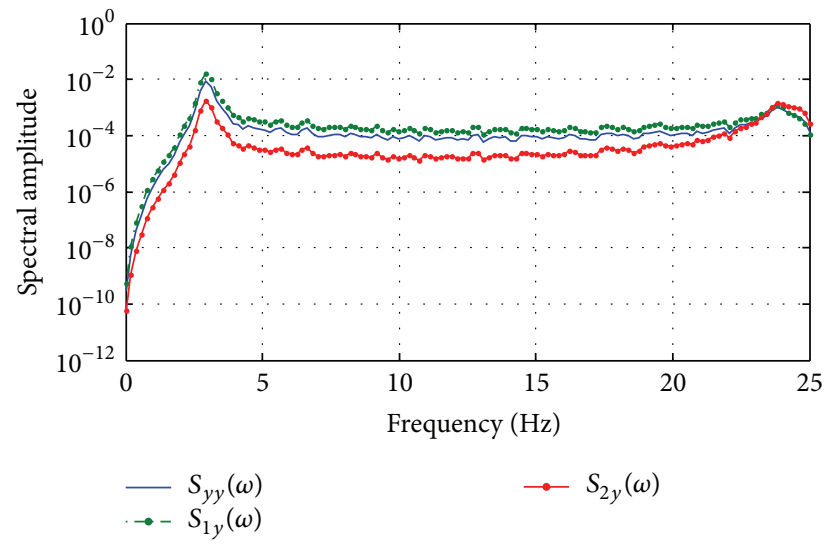

Figure 11: Power spectral density of substructural system 1. 


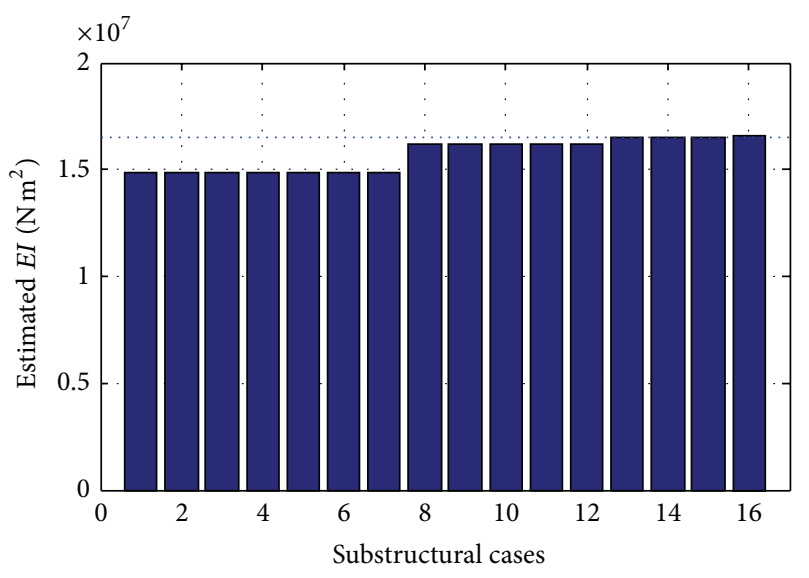

(a) Estimated EI values

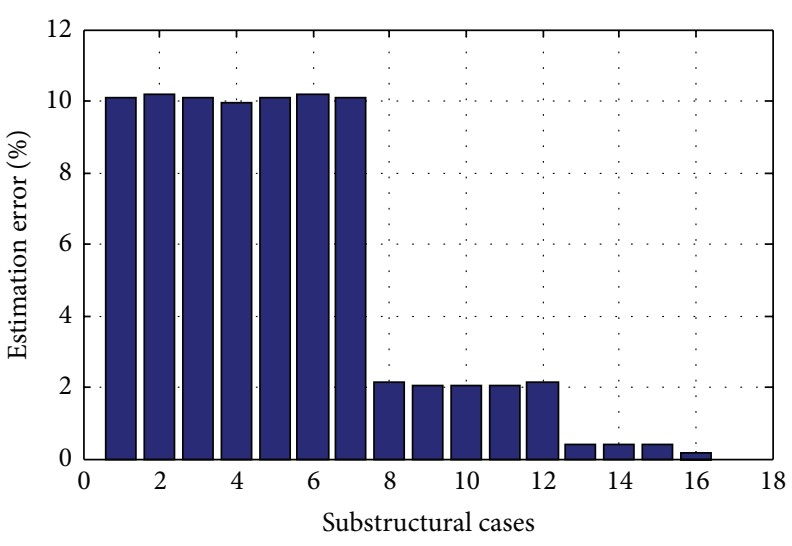

(b) Estimation errors

FIgURE 12: Estimation results (dotted line: exact EI value $=1.654 \times 10^{7} \mathrm{Nm}^{2}$ ).

reduced by increasing the length of an internal substructure. In the case of Substructural Cases 1-6 with a minimum length of substructure ( $2 \mathrm{~m}$ ), the estimation errors are mostly around $10 \%$, and the estimation errors are continuously reduced as low as about $2 \%, 0.5 \%$, and $0.1 \%$ as the substructure is getting longer and longer to $4 \mathrm{~m}, 6 \mathrm{~m}$, and $8 \mathrm{~m}$. This is because that the longer substructural behavior can follow much closely to the Bernoulli-Euler beam theory. And one more interesting observation can be obtained from the estimation errors in the results with the same substructural length, that is, among Cases 1-6, Cases 7-12, and Cases 13-15. The estimation errors for the cases with central substructures, that is, Substructural Case 4 among Cases 1-7, Substructural Case 10 among Cases 8-12, and Substructural Case 14 among Cases 13-15, are slightly less than the others. This means that the central part of whole structure is following closely to the Bernoulli-Euler beam theory when it is compared with other substructures with the same length.

\subsection{Application to Wind Turbine Tower Structure. The pro-} posed method is applied for identifying the flexural rigidity of a wind turbine tower structure. Recently wind turbine systems are getting more popularly utilized to extract clean energy from wind as much as possible and they are currently considered as one of the major civil infrastructures. Up to now, the tower structures are mostly damaged due to strong typhoons or failures in top side including rotor blades and drivetrain. However the structural integrity of a tower structure will be also one of the major concerns because towers are getting higher to utilize more stable and abundant wind resources and the lengthening is structurally unfavorable due to high possibility of buckling. The applicability of the proposed method is investigated using the numerical simulation data for a NREL 5MW wind turbine system as shown in Figure 13 [18]. This tower structure is assumed to be supported as a fixed condition at the bottom and the rotor and nacelle are simply modelled as lumped mass at the top of the tower. Two types of external loads are considered; the first is a random excitation with broadband frequency components and the second is a simulated thrust force obtained from FAST code [19], to investigate the effect of the type of an external load on the estimation accuracy. Even though the tower structure consists of several nonuniform sections in the NREL 5MW wind turbine model, the tower structure is herein considered to have a uniform section with area of $0.355 \mathrm{~m}^{2}$ and moment of inertia of $1.0705 \mathrm{~m}^{4}$ because the proposed method is formulated based on a Bernoulli-Euler beam theory with a uniform section. The mass density and elastic modulus are considered as $8,500 \mathrm{~kg} / \mathrm{m}^{3}$ and $247.2 \mathrm{GPa}$, respectively, as in the reference [18].

As shown in Figure 14, we divided the internal substructure into 5 different cases. For each case, the horizontal and rotational accelerations at the upper interfacial node are obtained using the numerical simulation results. And the flexural rigidity is estimated from the calculated acceleration responses.

Figure 15 shows the input and output acceleration responses at both interfaces and at the center of the internal substructure for Substructural Case 1. Figure 16 shows the PSD function of the vertical acceleration, $\ddot{d}_{2}(t)$, at the center of the internal substructure. The first natural frequency is obtained as $0.270 \mathrm{~Hz}$. As seen from the PSD of Substructural Case 1 under random and thrust force cases, the frequency contents are quite different; several excitation frequency components can be observed in the PSD plot for the case with thrust force excitation. In the case of ordinary modal identification problem, this kind of exciting frequency component is unfavorable for the modal identification. Figure 17 shows the estimated EI values for the cases under random excitation and thrust force excitation, and it can be easily observed that the results under random excitation are better than the results under thrust force excitation, which reveals that the exciting frequency components (or colored components) are acting as one of the adverse factors. In the cases of thrust force excitation, the estimation errors are fluctuated and it is relatively difficult to conclude that the longer substructure is better for accurate estimation. However the estimation results can be enhanced as longer substructure is utilized in the 


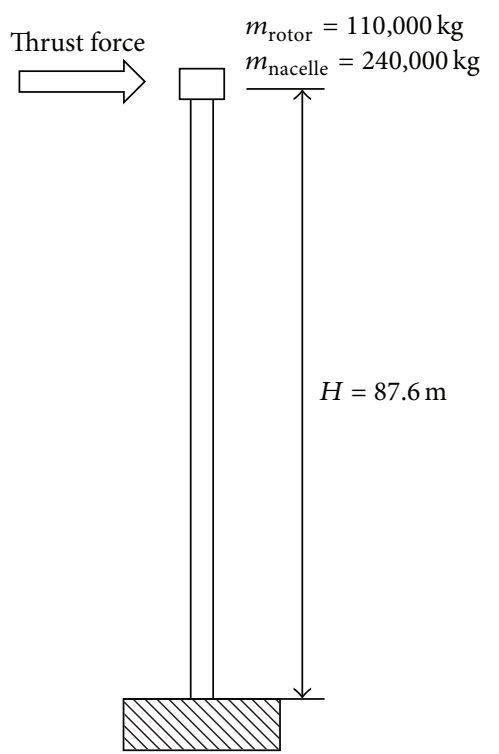

(a) Wind turbine tower
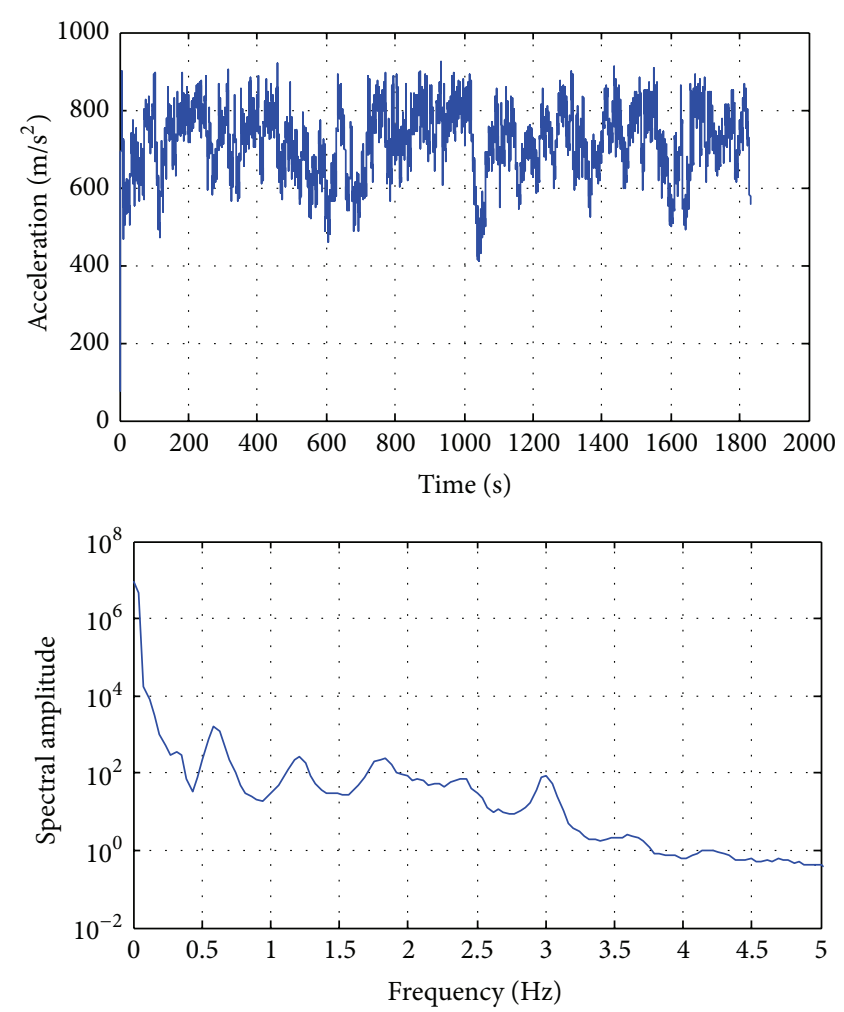

(b) Thrust force (top: time history, bottom: spectral amplitude)

FIGURE 13: Wind turbine tower and thrust force.

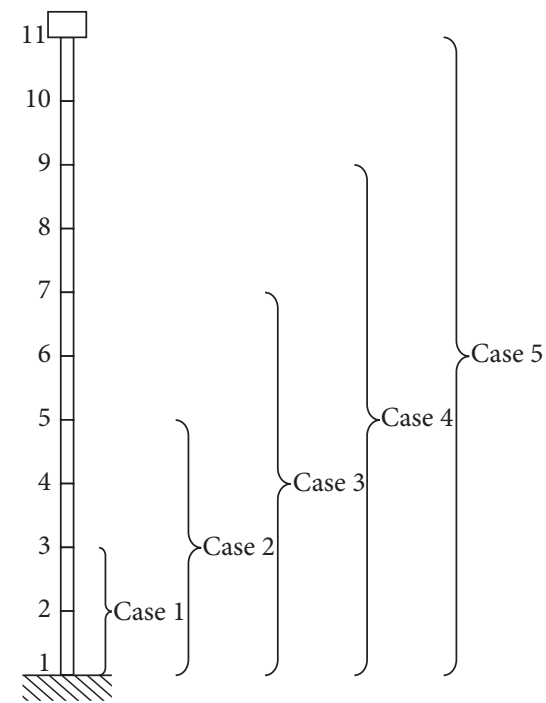

FIGURE 14: 3 Different cases for the substructural system.

cases of random excitations. It is noticed that the dynamic responses under low wind speed and/or before the rotor blade rotates would be better for identifying the flexural rigidity and also the impulse responses after emergency stop or break-on can be effectively utilized because the rotation of rotor blades makes a certain excitation frequency component which can make an adverse condition for identification.
3.4. Application to Steel Box-Girder Bridge Model: Experimental Case. In this example, the structural damages are artificially inflicted by loosening bolts between steel box blocks and the equivalent flexural rigidity of the superstructure is estimated using the proposed method (see Figure 18). Figure 19 shows the 6 damage scenarios. In Damage Case 1, the exterior bolts in the upper connecting plates between 


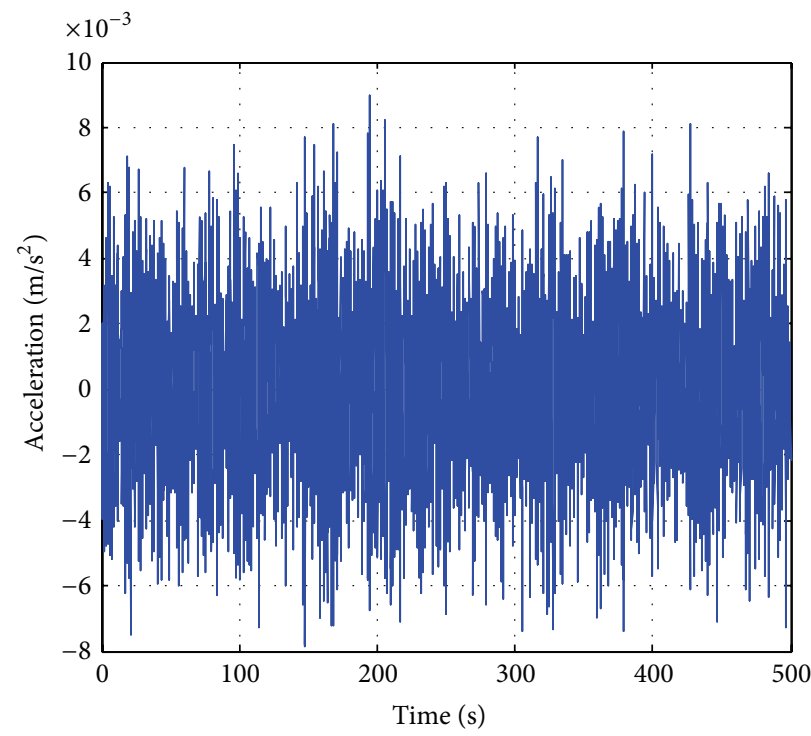

(a) $\ddot{u}_{1}(t)=\ddot{d}_{1}(t)+\ddot{d}_{3}(t)$

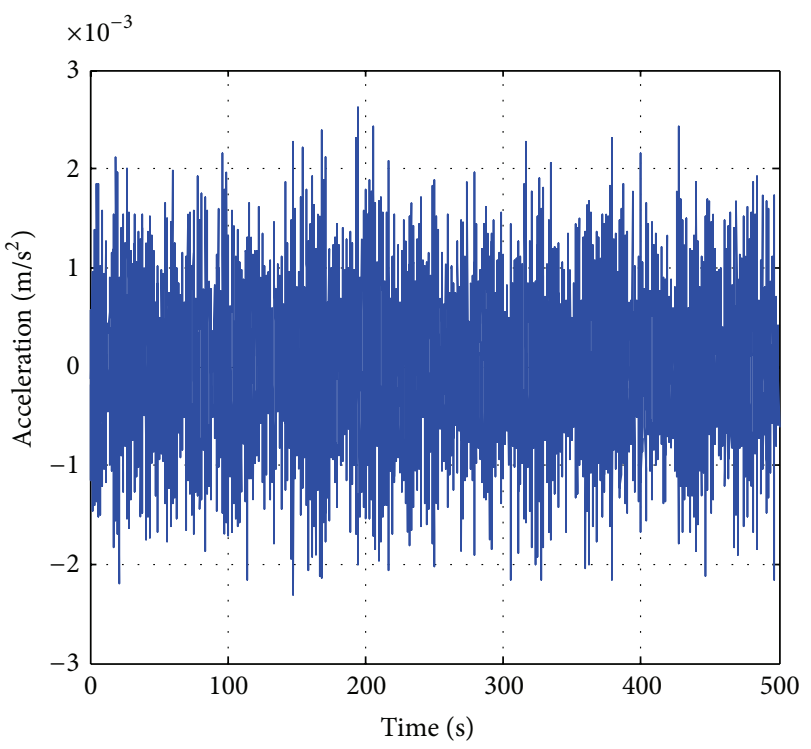

(b) $\ddot{d}_{2}(t)$

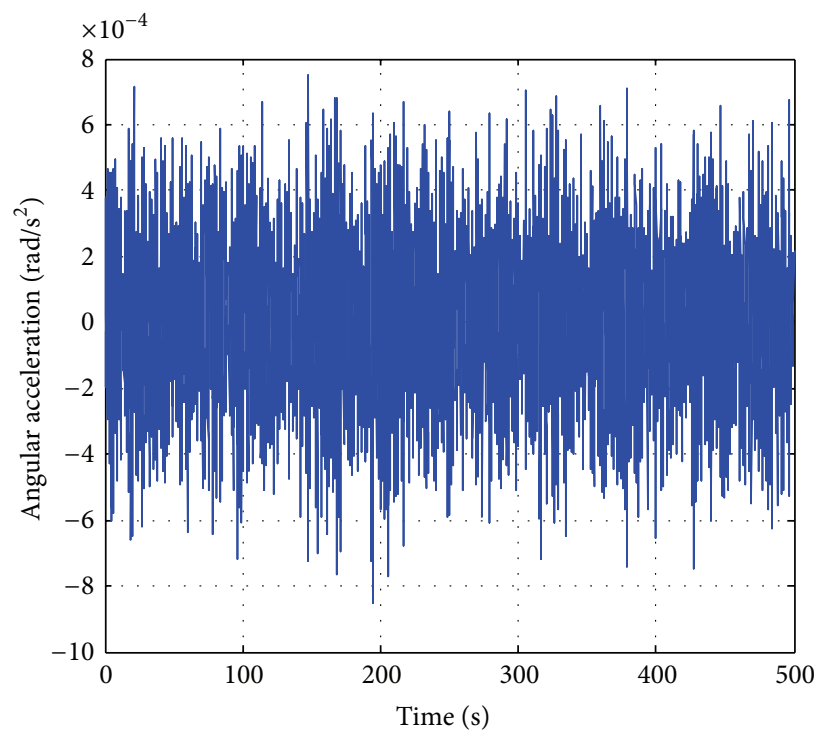

(c) $\ddot{u}_{2}(t)=\ddot{\phi}_{1}(t)-\ddot{\phi}_{3}(t)$

FIGURE 15: Input-output time histories of Substructural Case 1 under thrust force.

the second and the third blocks are loosened. In Damage Case 2, all the bolts are loosened in the same damaged plate for Damage Case 1. In Damage Case 3, the two rows of the side connecting plates are additionally loosened, and in Damage Case 4 all the bolts are loosened in the upper plate between the third and fourth blocks additionally. In Damage Case 5, the bolts in the upper plate between the fourth and fifth blocks are loosened instead of the upper plate between the third and fourth blocks (in Damage Case 4). In Damage Case 6, the bolts in the upper plate and the side plate connecting the second and third blocks are tightened, and this can be considered as the partially repaired condition.

The internal substructure is defined as shown in Figure 20 to estimate the equivalent flexural rigidity of a damaged beam. The mean acceleration of accelerations from two adjacent sensors is used as vertical acceleration $\left(\ddot{d}_{1}(t)\right.$ and $\ddot{d}_{3}(t)$ ) while the difference is used to calculate rotational acceleration $\left(\ddot{\phi}_{1}(t)\right.$ and $\left.\ddot{\phi}_{3}(t)\right)$ as shown in Figure 21. PSD function of acceleration response at the center of the internal substructure is obtained as shown in Figure 22. The vertical and rotational responses are approximately obtained by averaging and differencing the responses measured from the adjacent two sensors.

The estimated results for 6 damage cases are summarized in Table 3 and the estimated flexural rigidity is gradually decreased as the structural damage is getting more severe from Damage Case 1 to Damage Cases 2, 3, 4, and 5. However flexural rigidities are slightly increased from Damage Case 2 


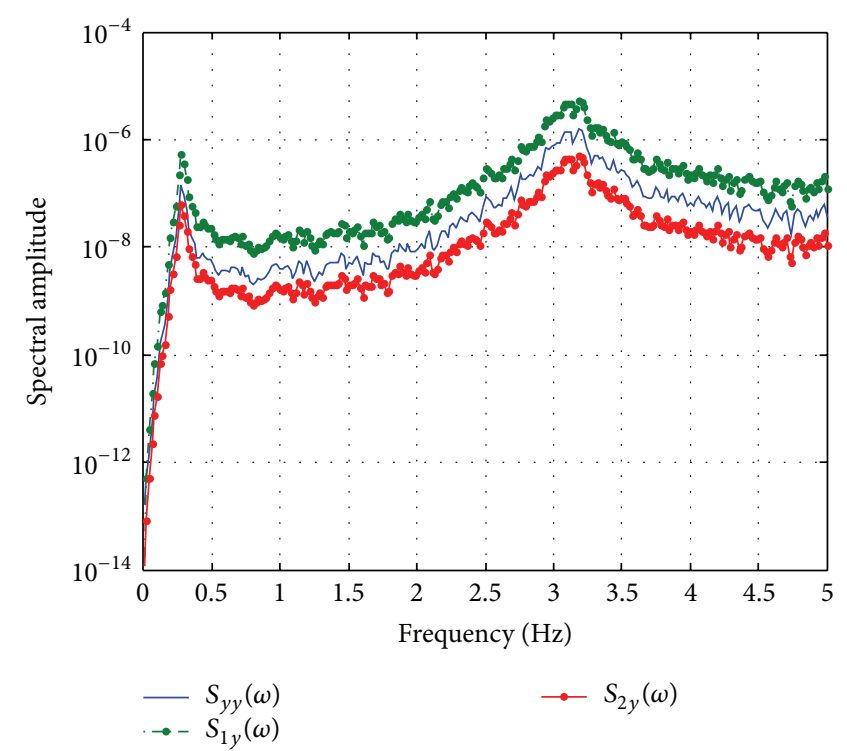

(a) Random excitation

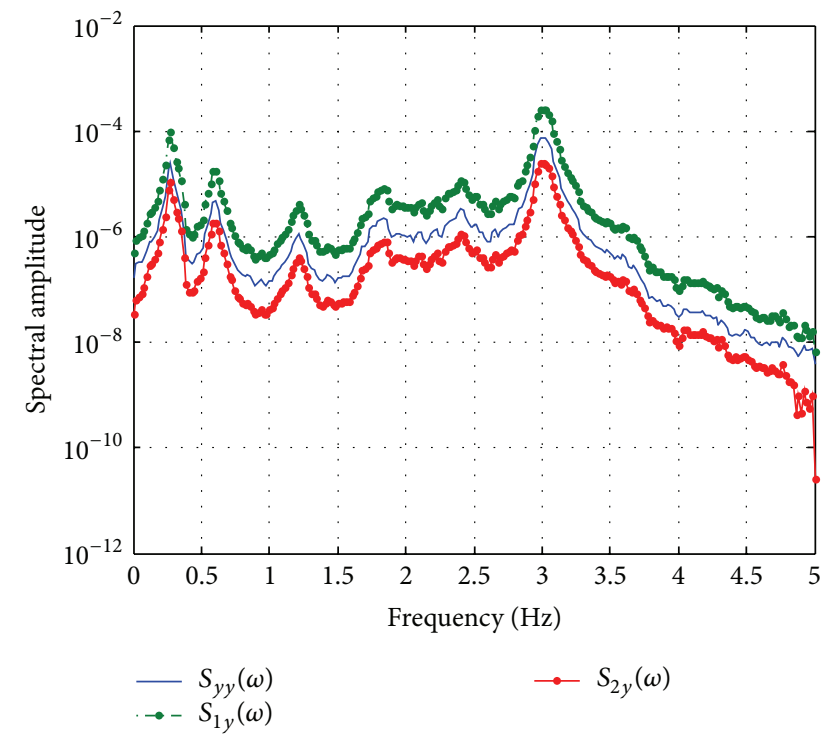

(b) Thrust force

Figure 16: Power spectral density of Substructural Case 1.
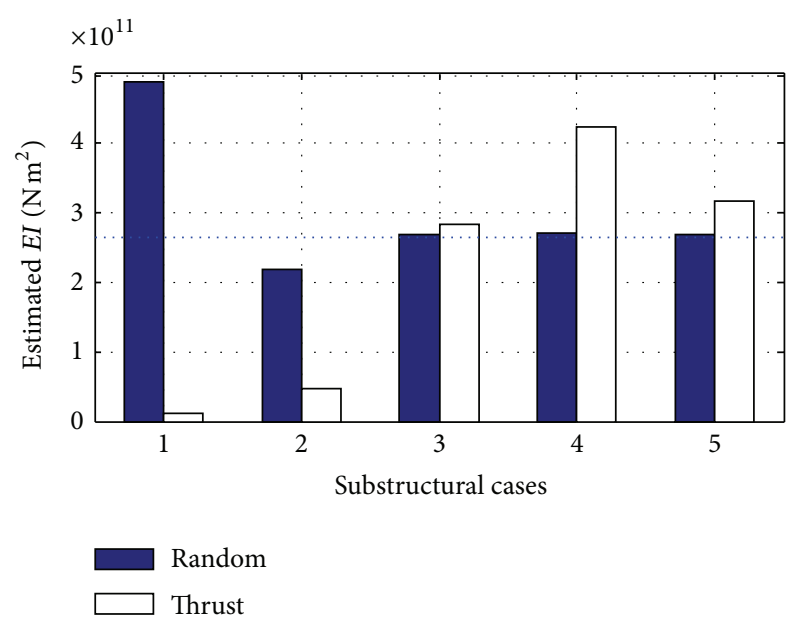

FIGURE 17: Estimated results (dotted line: exact $E I$ value $=2.646 \times$ $10^{11} \mathrm{Nm}^{2}$ ).

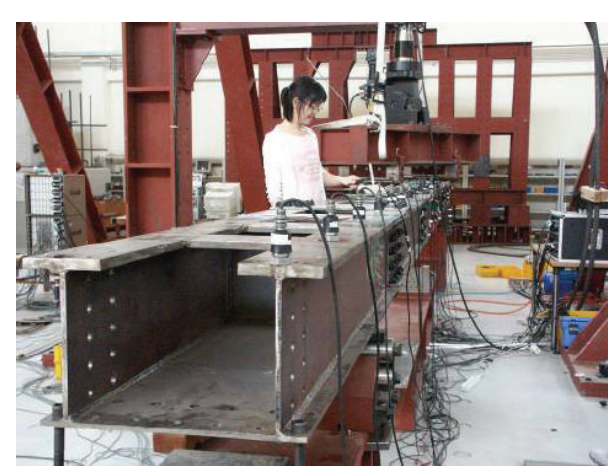

FIGURE 18: Overview of experimental tests.
TABLE 3: Estimated flexural rigidity for each damage case.

\begin{tabular}{lcccc}
\hline Damage cases & $f_{1}(\mathrm{~Hz})$ & $\xi$ & $E I(\mathrm{Nm})$ & $\left(E I_{i} / E I_{o}\right)-1(\%)$ \\
\hline Intact & 13.245 & 1.142 & $1.51 \times 10^{7}$ & 0.00 \\
1 & 13.123 & 1.666 & $1.37 \times 10^{7}$ & -9.27 \\
2 & 12.695 & 1.666 & $1.28 \times 10^{7}$ & -15.23 \\
3 & 12.299 & 1.145 & $1.29 \times 10^{7}$ & -14.57 \\
4 & 11.932 & 1.142 & $1.23 \times 10^{7}$ & -18.54 \\
5 & 12.207 & 1.651 & $1.25 \times 10^{7}$ & -17.22 \\
6 & 13.153 & 1.657 & $1.42 \times 10^{7}$ & -5.96 \\
\hline
\end{tabular}

to Damage Case 3 by about $0.8 \%$ and it is expected that the measurement noise and approximation errors affect the accuracy of the estimation. Damage Case 4 and Damage Case 5 are almost the same and the estimated results also show that two damages are not significantly deviated. For Damage Case 6, since the bolts in the connecting plates between the second and third blocks are tightened again, it is expected that the flexural rigidity is increased. The results show that the flexural rigidity is increased from $12.5 \mathrm{MNm}$ to 14.2 $\mathrm{MNm}$ and this estimated flexural rigidity is lower than that of intact case and greater than that of Damage Case 1, even though the Damage Case 1 is more slight damage than Damage Case 6, which means the damage in side connecting part is not more severe than the structural damage in central part.

\section{Conclusions}

A new substructural identification method is proposed to estimate the flexural rigidity of a beam-like structure such as bridge deck without consideration on the actual boundary conditions in service. The proposed method is fairly simple 


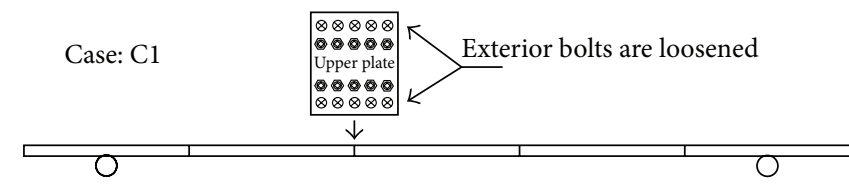

(a) Damage Case 1

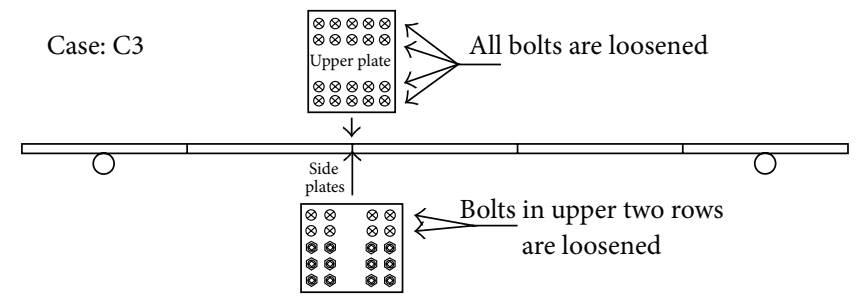

(c) Damage Case 3

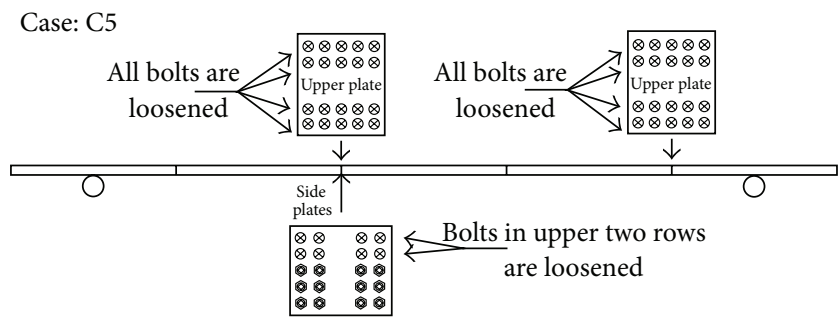

(e) Damage Case 5

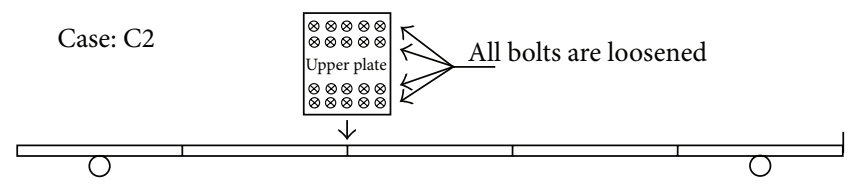

(b) Damage Case 2

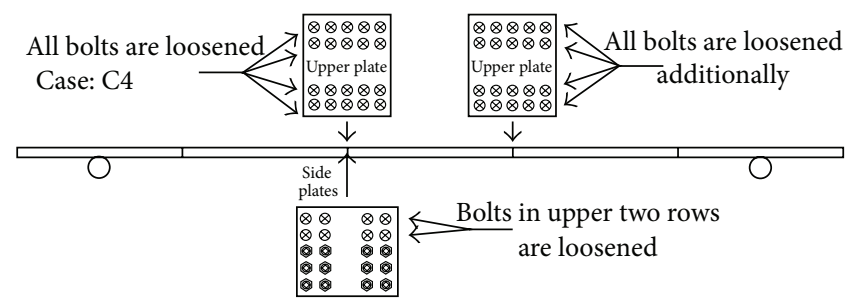

(d) Damage Case 4

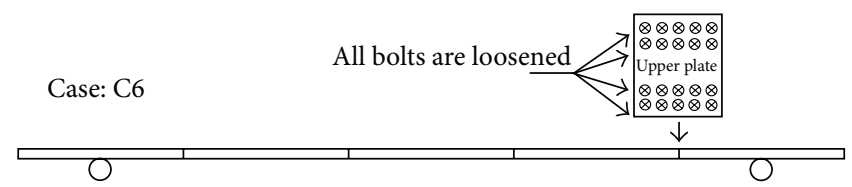

(f) Damage Case 6

FiguRE 19: Inflicted damage scenarios.

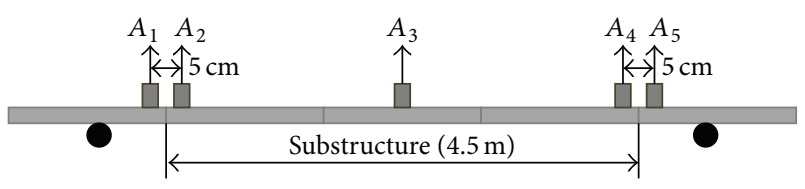

FIGURE 20: Substructure used for estimating flexural rigidity.

and effective since it requires only five acceleration measurements around the internal substructure and the single variable optimization scheme. The performance of the proposed method is verified by estimating the flexural rigidity of a beam-like structure with different boundary conditions by experimental study using a simple beam model. It is also verified that the proposed method can estimate the flexural rigidity regardless of the definition of internal substructure through numerical study using a concrete plate-girder bridge model. However it is also found that the estimation results can be enhanced by using longer internal substructure because the dynamic behavior of a longer internal substructure follows much more closely to the Bernoulli-Euler beam theory. The effect of the excitation type is also investigated through a cantilever-type wind turbine tower structure, and it is found that the random excitation with broadband frequency components is better for the reliable identification. Therefore it is recommended to use the dynamic responses under low wind speed before the rotor blade rotates and/or after emergency stop or break-on because the rotation of rotor blade makes a certain excitation frequency component which can make an adverse condition for identification. Finally, the equivalent flexural rigidities of the damaged structures can be easily estimated by the proposed method.

For the successful application of the proposed method, it is crucial to measure the rotational responses, and it is obtained by introducing two sensors at the adjacent locations in this study. It can be more easily obtained in the near future owing to the advanced dynamic inclinometers; therefore this method can become much more feasible to identify the flexural rigidity of beam-like structures such as longspan bridges, high-rise buildings, and also very-tall wind towers. For choosing the most appropriate inclinometers, the effects of measurement performance including accuracy and precision may be investigated in the further study.

It is also noticed that there still exist several limitations for the successful real application of the proposed method including the following: (1) the proposed method can be effectively utilized to identify the flexural rigidity of a beamlike structure where pure bending modes can be separated from shear and torsional vibration modes, (2) this method is developed based on the Bernoulli-Euler beam theory with uniform section; therefore the identification results might be inaccurate when the internal substructural section is nonuniform, and (3) the external mass effects such as moving vehicles were not considered in formulation; therefore the dynamic responses after passing away of moving vehicles are preferable for reasonable identification by removing the external mass effects. Further studies can be carried out to resolve these limitations and also it is recommended to extend this method to combine other newly proposed approaches and data processing techniques including ant colony optimization [20] and wavelet transform [21, 22]. 

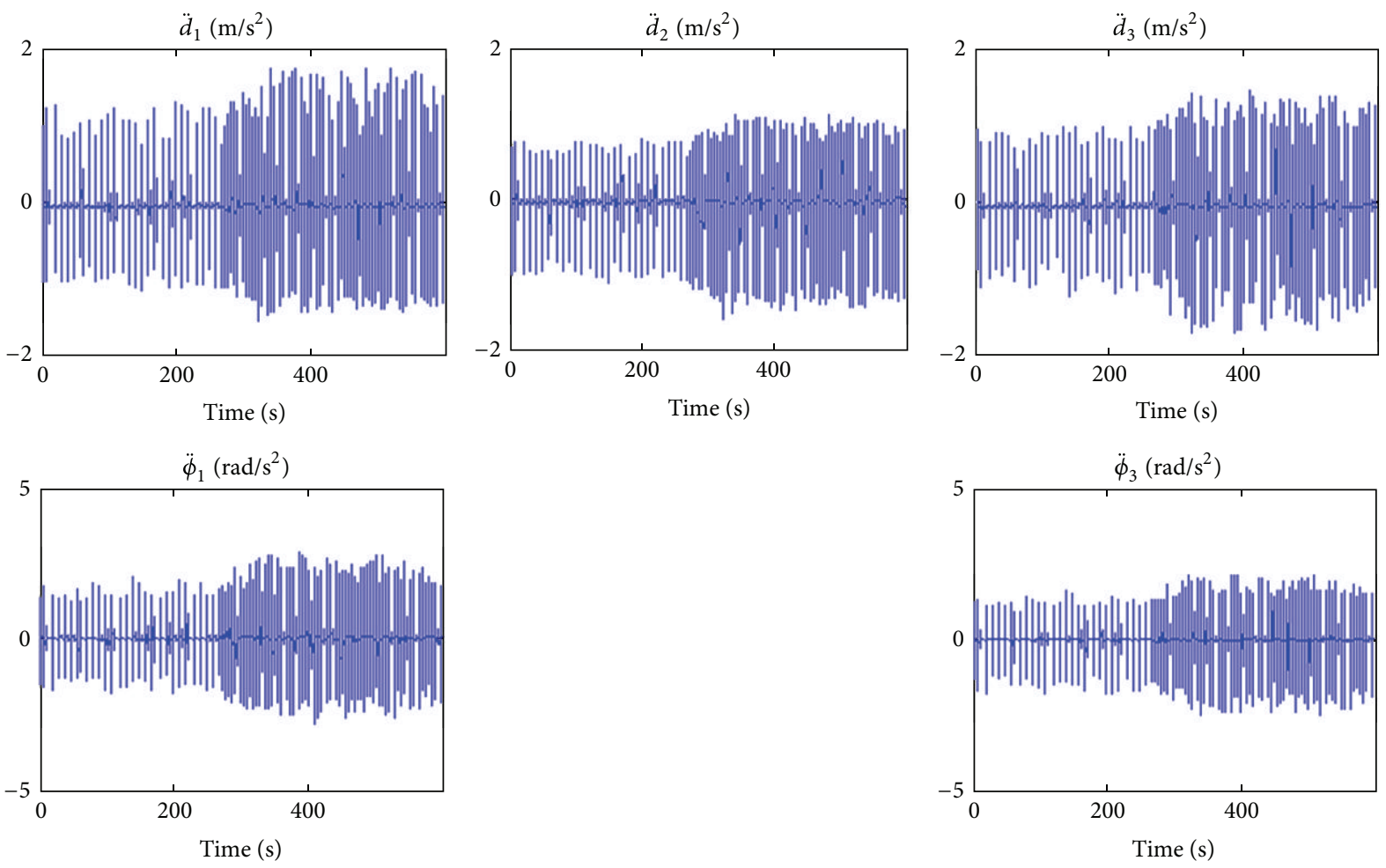

FIGURE 21: Input and output responses for prescribed substructural system.

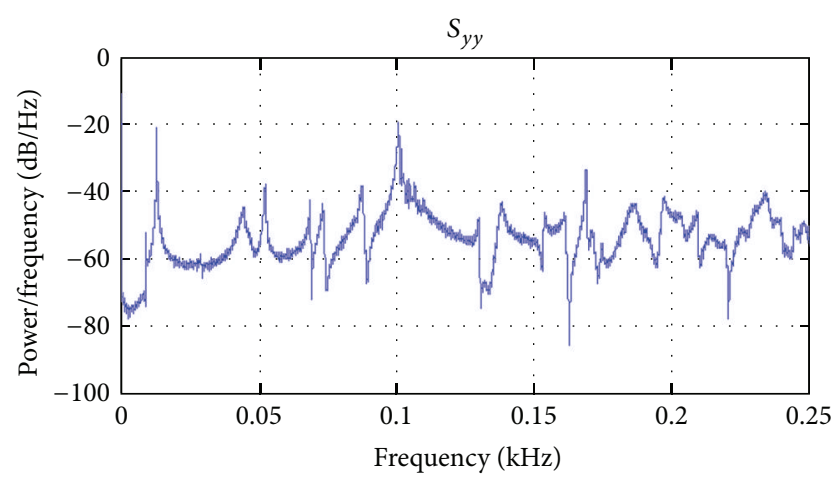

FIGURE 22: PSD of output responses at the center of substructure.

\section{Conflict of Interests}

The authors declare that there is no conflict of interests regarding the publication of this paper.

\section{Acknowledgment}

This research was financially supported by the Ministry of Land, Infrastructure and Transport (MOLIT) of the Korea government (code 12 Technology Innovation E09). This study is an extended work of the authors' previous one, Koo et al. [23].

\section{References}

[1] L. Yu and T. Yin, "Damage identification in frame structures based on FE model updating," Journal of Vibration and Acoustics, Transactions of the ASME, vol. 132, no. 5, 13 pages, 2010.

[2] L. Yu, J.-H. Zhu, and L.-L. Yu, "Structural damage detection in a truss bridge model using fuzzy clustering and measured FRF data reduced by principal component projection," Advances in Structural Engineering, vol. 16, no. 1, pp. 207-217, 2013.

[3] H.-N. Li, T.-H. Yi, L. Ren, D.-S. Li, and L.-S. Huo, "Reviews on innovations and applications in structural health monitoring for infrastructures," Structural Monitoring and Maintenance, vol. 1, no. 1, pp. 1-45, 2014.

[4] C.-H. Min, J.-S. Choi, S. Hong, H.-W. Kim, and T.-K. Yeu, "Damage detection using finite element model updating," Journal of Ocean Engineering and Technology, vol. 26, no. 5, pp. 11-17, 2012.

[5] S.-Y. Lee, S.-R. Lee, and J.-T. Kim, "Experimental modal analysis for damage identification in foundation-structure interface of Caisson-type breakwater," Journal of Ocean Engineering and Technology, vol. 26, no. 1, pp. 34-40, 2012.

[6] H. Sohn, M. Dzwonczyk, E. G. Straser, A. S. Kiremidjian, K. Law, and T. Meng, "An experimental study of temperature effect on modal parameters of the Alamosa Canyon Bridge," Earthquake Engineering and Structural Dynamics, vol. 28, no. 7-8, pp. 879-897, 1999.

[7] S. Alampalli, "Effects of testing, analysis, damage, and environment on modal parameters," Mechanical Systems and Signal Processing, vol. 14, no. 1, pp. 63-74, 2000. 
[8] D.-S. Hong and J.-T. Kim, “Temperature effect on impedancebased damage monitoring of steel-bolt connection using wireless impedance sensor node," Journal of Ocean Engineering and Technology, vol. 26, no. 1, pp. 27-33, 2012.

[9] A. W. C. Oreta and T.-A. Tanabe, "Element identification of member properties of framed structures," Journal of Structural Engineering, vol. 120, no. 7, pp. 1961-1976, 1994.

[10] C.-B. Yun and H.-J. Lee, "Substructural identification for damage estimation of structures," Structural Safety, vol. 19, no. 1, pp. 121-140, 1997.

[11] C. G. Koh, B. Hong, and C. Y. Liaw, "Substructural and progressive structural identification methods," Engineering Structures, vol. 25, no. 12, pp. 1551-1563, 2003.

[12] C. G. Koh and K. Shankar, "Substructural identification method without interface measurement," Journal of Engineering Mechanics, vol. 129, no. 7, pp. 769-776, 2003.

[13] Z. Zhang, C. G. Koh, and M. J. Perry, "Frequency domain substructural identification for arbitrary excitations," Earthquake Engineering and Structural Dynamics, vol. 41, no. 4, pp. 605-621, 2012.

[14] J. Li, S. S. Law, and Y. Ding, "Substructure damage identification based on response reconstruction in frequency domain and model updating," Engineering Structures, vol. 41, pp. 270-284, 2012.

[15] J. Li and S. S. Law, "Damage identification of a target substructure with moving load excitation," Mechanical Systems and Signal Processing, vol. 30, pp. 78-90, 2012.

[16] S. Weng, H.-P. Zhu, Y. Xia, and L. Mao, "Damage detection using the eigenparameter decomposition of substructural flexibility matrix," Mechanical Systems and Signal Processing, vol. 34, no. 1-2, pp. 19-38, 2013.

[17] S. Weng, Y. Xia, X.-Q. Zhou, Y.-L. Xu, and H.-P. Zhu, "Inverse substructure method for model updating of structures," Journal of Sound and Vibration, vol. 331, no. 25, pp. 5449-5468, 2012.

[18] J. Jonkman, S. Butterfield, W. Musial, and G. Scott, "Definition of a 5-MW reference wind turbine for offshore system development," Tech. Rep. NREL/TP-500-38060, National Renewable Energy Laboratory, 2009.

[19] J. M. Jonkman and M. L. Buhl, "FAST user's guide," Tech. Rep. NREL/EL-500-38230, 2005.

[20] L. Yu and P. Xu, "Structural health monitoring based on continuous ACO method," Microelectronics Reliability, vol. 51, no. 2, pp. 270-278, 2011.

[21] H. Li, T. Yi, M. Gu, and L. Huo, "Evaluation of earthquakeinduced structural damages by wavelet transform," Progress in Natural Science, vol. 19, no. 4, pp. 461-470, 2009.

[22] T.-H. Yi, H.-N. Li, and M. Gu, "Wavelet based multi-step filtering method for bridge health monitoring using GPS and accelerometer," Smart Structures and Systems, vol. 11, no. 4, pp. 331-348, 2013.

[23] K.-Y. Koo, J.-H. Yi, and C.-B. Yun, "Structural integrity assessment of beam type structures using substructural identification technique," Journal of the Korean Society of Civil Engineers, vol. 27, no. 5A, pp. 691-699, 2007 (Korean). 

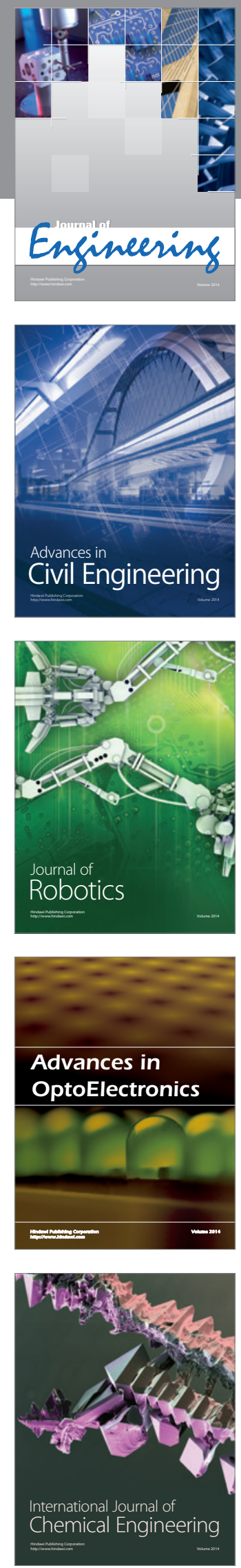

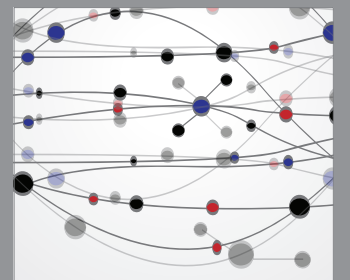

The Scientific World Journal
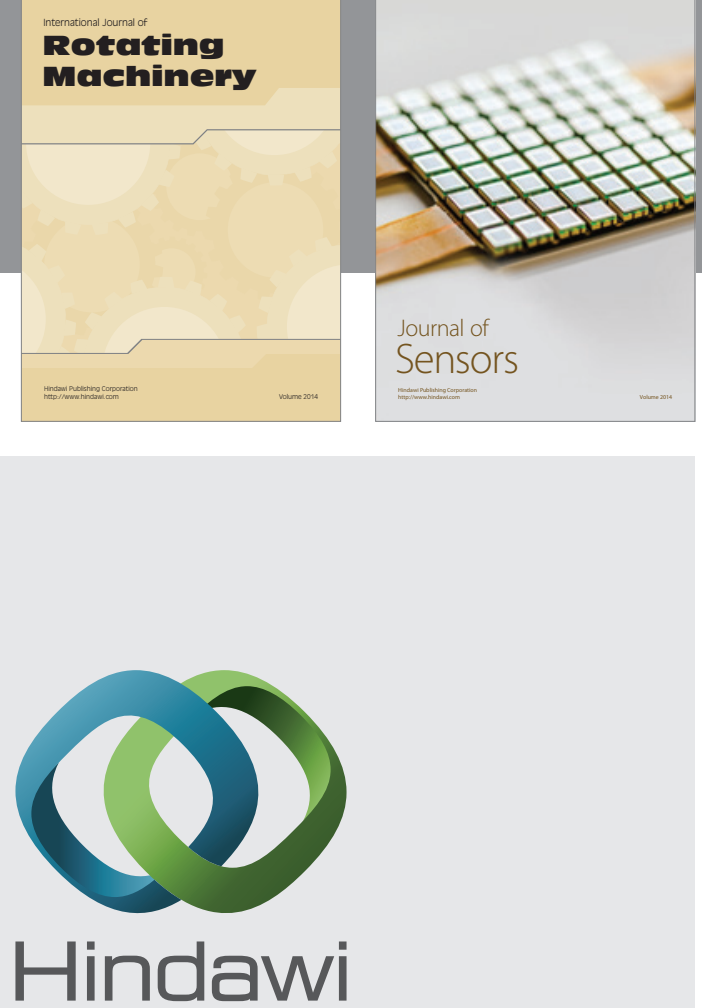

Submit your manuscripts at http://www.hindawi.com
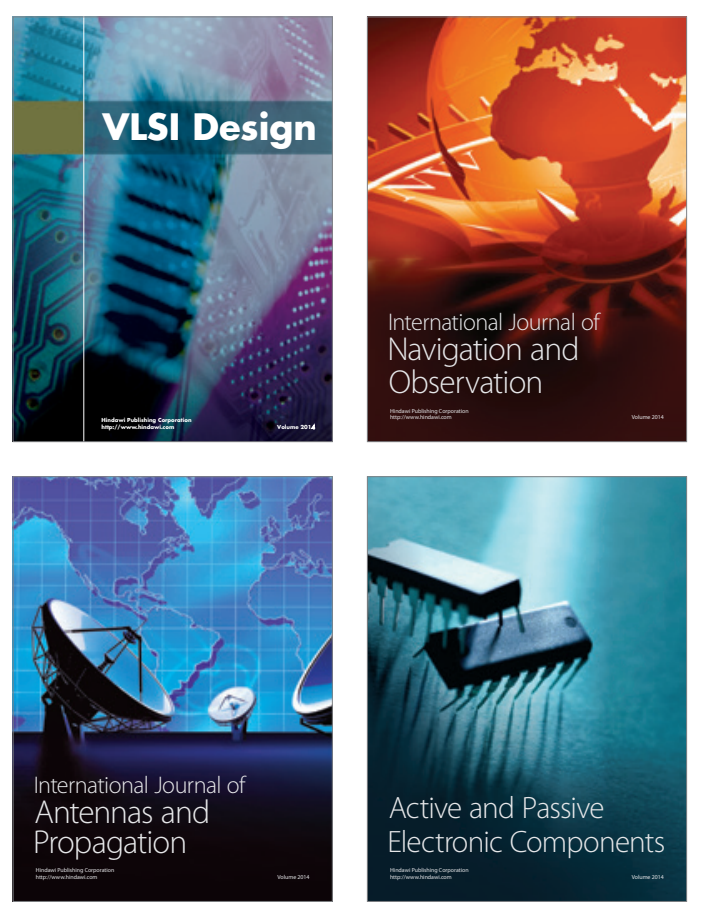
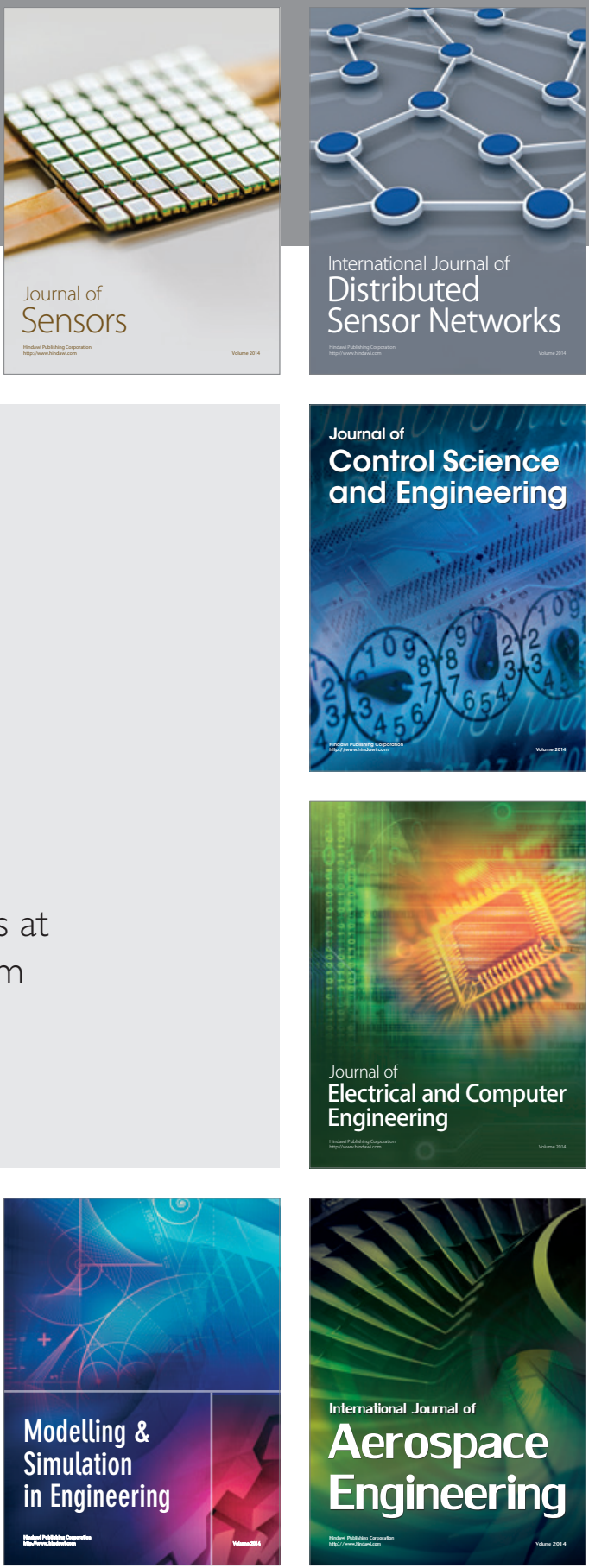

Journal of

Control Science

and Engineering
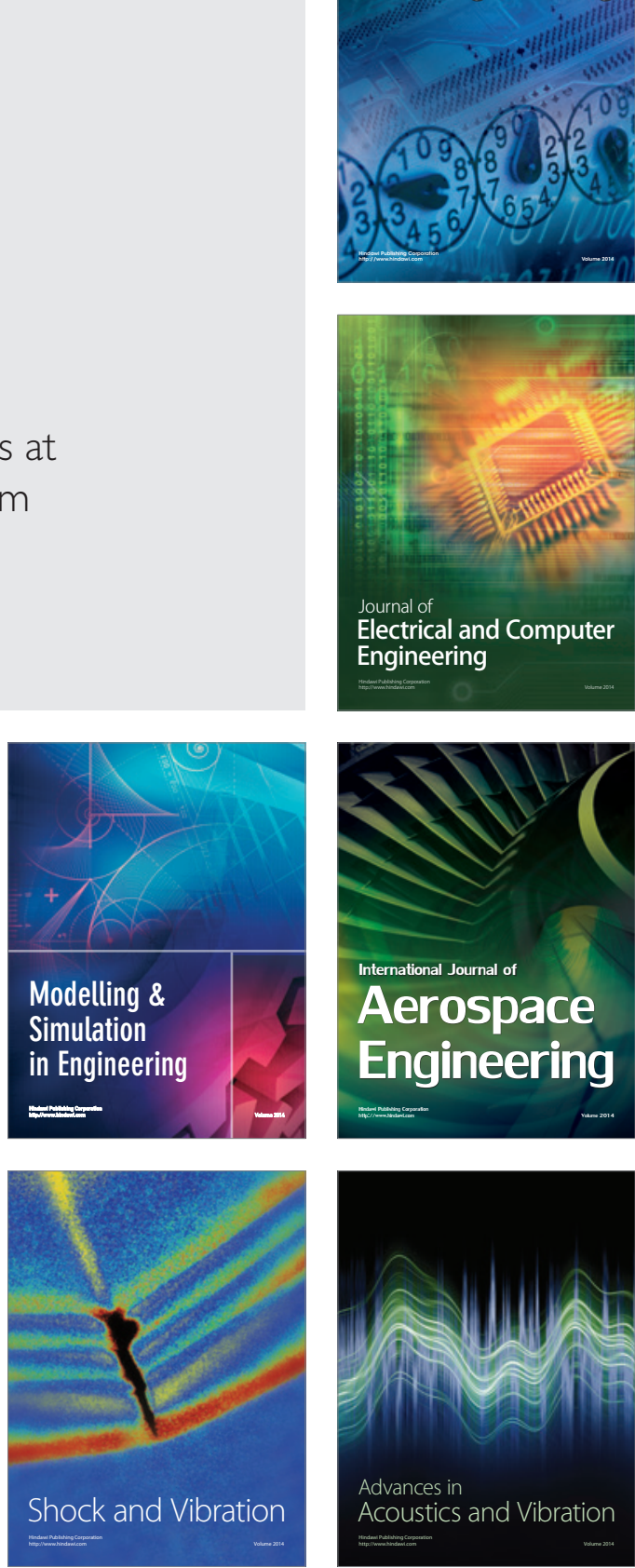